Article

\title{
Biochemical and Comparative Transcriptome Analyses Reveal Key Genes Involved in Major Metabolic Regulation Related to Colored Leaf Formation in Osmanthus fragrans 'Yinbi Shuanghui' during Development
}

\author{
Xuan Chen ${ }^{1,2}$, Xiulian Yang ${ }^{1,3}$, Jun Xie ${ }^{2}$, Wenjie Ding ${ }^{1,3}$, Yuli Li ${ }^{1,3}$, Yuanzheng Yue ${ }^{1,3, *}$ and \\ Lianggui Wang 1,3,*(D) \\ 1 Key Laboratory of Landscape Architecture, Jiangsu Province, College of Landscape Architecture, \\ Nanjing Forestry University, No. 159 Longpan Road, Nanjing 210037, China; chenxuan@njts.edu.cn (X.C.); \\ xly@njfu.edu.cn (X.Y.); wenjieding@njfu.edu.cn (W.D.); chestnutlyl@njfu.edu.cn (Y.L.) \\ 2 College of Fine Arts, Nanjing Normal University of Special Education, No.1 Shennong Road, \\ Nanjing 210038, China; xiejun@njts.edu.cn \\ 3 Co-Innovation Center for Sustainable Forestry in Southern China, Nanjing Forestry University, \\ Nanjing 210037, China \\ * Correspondence: yueyuanzheng@njfu.edu.cn (Y.Y.); wlg@njfu.com.cn (L.W.); Tel.: +86-138-0900-7625 (L.W.)
}

Received: 25 February 2020; Accepted: 1 April 2020; Published: 4 April 2020

\begin{abstract}
Osmanthus fragrans 'Yinbi Shuanghui' not only has a beautiful shape and fresh floral fragrance, but also rich leaf colors that change, making the tree useful for landscaping. In order to study the mechanisms of color formation in O. fragrans 'Yinbi Shuanghui' leaves, we analyzed the colored and green leaves at different developmental stages in terms of leaf pigment content, cell structure, and transcriptome data. We found that the chlorophyll content in the colored leaves was lower than that of green leaves throughout development. By analyzing the structure of chloroplasts, the colored leaves demonstrated more stromal lamellae and low numbers of granum thylakoid. However, there was a large number of plastoglobuli. Using transcriptome sequencing, we demonstrated that the expression of differentially expressed genes (DEGs) involved in chlorophyll degradation was upregulated, i.e., heme oxygennase-1 (HO1), pheophorbide a oxidase (PAO), and chlorophyllase-2 (CLH2), affecting the synthesis of chlorophyll in colored leaves. The stay-green gene (SGR) was upregulated in colored leaves. Genes involved in carotenoid synthesis, i.e., phytoene synthase 1 (PSY1) and 1-Deoxyxylulose-5-phosphate synthase (DXS), were downregulated in colored leaves, impeding the synthesis of carotenoids. In the later stage of leaf development, the downregulated expression of Golden2-Like (GLK) inhibited chloroplast development in colored leaves. Using weighted gene co-expression network analysis (WGCNA) to investigate the correlation between physiological indicators and DEGs, we chose the modules with the highest degree of relevance to chlorophyll degradation and carotenoid metabolism. A total of five genes (HSFA2, NFYC9, TCP20, WRKY3, and WRKY4) were identified as hub genes. These analyses provide new insights into color formation mechanisms in $O$. fragrans 'Yinbi Shuanghui' leaves at the transcriptional level.
\end{abstract}

Keywords: leaf color; transcriptome; chlorophyll metabolism; carotenoid metabolism

\section{Introduction}

Leaf color mutants exist widely in higher plants and are ideal materials for the study of physiological metabolic processes such as plant photosynthesis, photochemical function, phytohormones, and disease 
resistance mechanisms. These mutants can also be analyzed to identify gene functions and investigate inter-gene interactions. To date, researchers have obtained these mutants from crops such as Oryza sativa [1], Triticum aestivum [2], Arabidopsis thaliana [3], and Brassica napus [4]. A large number of studies have been carried out on pigment content metabolism [5], chloroplast development [6], photosynthetic physiology [7], genetic patterns [8], gene cloning [9,10], etc. These studies provide important basic information that aided the understanding of the mechanisms that underlie leaf color mutations and related gene function.

The direct causes of leaf color mutations include the changes in leaf pigment content and proportion, cell structure, and physiological and biochemical metabolism. The mechanism underlying leaf color variation is complex and mainly related to chlorophyll, carotenoid, secondary metabolite synthesis, photosynthesis, and chloroplast development. Remarkably, the leaf color changes in the leaf mutant and senescence process are usually related to the chlorophyll metabolism pathway [11,12]. However, the change in leaf color during leaf senescence is later followed by leaf drop, and the leaf color in coloration mutants start with the initial stage of leaf color formation and would keep this color during leaf growth and development. Studies on leaf color mutants in different varieties of Camellia sinensis show that patterns of differentially expressed genes (DEGs) involved in chlorophyll biosynthesis or degradation, carotenoid biosynthesis or degradation, and chloroplast development affect leaf color $[13,14]$. Compounds related to carotenoid and chlorophyll synthesis have confirmed that the $\mathrm{H}$ subunit of magnesium chelatase $(\mathrm{CHLH})$ and $\beta$-carotene hydroxylase $(\mathrm{BCH})$ are important to the abnormal leaf color phenotype of the T. aestivum yellow-green leaf mutant [15]. Studies on leaf color mutant of Cymbidium sinense 'Dharma' have demonstrated that the gene encoding key enzyme of chlorophyll degradation is expressed at a higher level, which is consistent with its lower chlorophyll content. It suggests that leaf color variation may be due to the excessive degradation of chlorophyll [16]. In addition, the upregulated expression of genes involved in flavonoid biosynthesis and the downregulation of genes involved in carotenoid biosynthesis may be related to the yellow-leaf phenotype [17]. A previous study has suggested that the color formation mechanism of the yellow leaf mutant of Lagerstroemia indica is influenced by chloroplast development and chlorophyll metabolism [18]. Additionally, the expression of polyphenol oxidase (PPO) and non-yellow coloring (NYC/NOL) may affect chlorophyll biosynthesis in Ginkgo biloba yellow-green mutants and promote chlorophyll b degradation to chlorophyll a. The upregulated expression of 15 -cis- $\zeta$-carotene isomerase (Z-ISO), $\zeta$-carotene desaturase (ZDS), and lycopene $\varepsilon$-cyclase (LCYE) enhance carotenoid accumulation [19].

$O$. fragrans is one of the top ten traditional ornamental crops native to China. The tree offers not only a beautiful shape and the fresh floral fragrance of traditional sweet osmanthus, but also rich leaf color changes, making it a good material for landscaping [20]. During diversification, many traits have been mutated due to natural hybridization, manual selection, and other environmental factors. Also, the number of varieties is becoming greater. $O$. fragrans is divided into five varieties: the Aurantiacus, Albus, Asiaticus, Luteus, and Color groups [21]. O. fragrans 'Yinbi Shuanghui' is derived from the Asiaticus group, which has a limbate leaf with a yellow-white margin and green in the middle.

In order to investigate the mechanisms of leaf color formation in 'Yinbi Shuanghui', we selected colored and green leaves from the same plant and analyzed leaf pigment contents, cell structures, and transcriptome data at different developmental stages. By comparing transcriptome data, we identified gene expression patterns related to chlorophyll and carotenoid metabolism. Weighted gene co-expression network analysis (WGCNA) was used to analyze the correlation between physiological indicators and DEGs. We then identified hub genes involved in regulating chlorophyll and carotenoid metabolic pathways at different developmental stages. This study provides a reference that could be used to investigate the color formation mechanisms of leaf color mutants. 


\section{Material and Methods}

\subsection{Plant Materials}

A healthy adult $O$. fragrans 'Yinbi Shuanghui' tree bearing both colored leaves $(C)$ and green leaves $(G)$ was used for transcriptome analysis. The plants were 3 years old and had been grown in the field at Liyang (N 31 $43^{\prime}$, E $119^{\circ} 48^{\prime}$ ), China. 'Yinbi Shuanghui' was a colored-leaf cultivar that was evolved from $O$. fragrans 'Sijigui'. Three developmental stages for leaf color in 'Yinbi Shuanghui' were identified: the young leaf stage (A), leaf-expanding stage (B), and mature leaf stage (C). We collected leaf samples from colored and green leaves at three different stages, respectively, to measure the pigment content and cell structure, as well as for transcriptome sequencing analysis. All samples were collected at $10 \mathrm{am}$, transferred immediately to liquid nitrogen, and stored subsequently at $-80^{\circ} \mathrm{C}$ until RNA extraction. Leaves at each stage were sampled from three comparable plants using three biological replications. The samples collected at stages A, B, and C were used to construct 18 libraries.

\subsection{Pigment Determination}

Pigments were extracted from leaves using the methods described by Zhang [22]. Chlorophyll and carotenoids were extracted with $95 \%$ ethanol for $24 \mathrm{~h}$ and quantified spectrophotometrically (Lambda 365, PerkinElmer, USA) at 470, 645, and $663 \mathrm{~nm}$. Anthocyanin was extracted with 10\% hydrochloric acid in darkness for $24 \mathrm{~h}$ and quantified spectrophotometrically at $530 \mathrm{~nm}$.

\subsection{Transmission Electron Microscopy}

Samples dissected from yellow and green parts of colored leaves and green leaves were cut into smaller sections that were approximately $1.0 \mathrm{~mm} \times 1.0 \mathrm{~mm} \times 1.0 \mathrm{~mm}$ in size. The samples were fixed in $4 \%$ glutaraldehyde for $24 \mathrm{~h}$ at $4{ }^{\circ} \mathrm{C}$, washed twice with $0.1 \mathrm{M}$ phosphate buffer for 10 min each time, then fixed in $1 \% \mathrm{OsO}_{4}$ for $5 \mathrm{~h}$, and then washed twice with $0.1 \mathrm{M}$ phosphate buffer for $10 \mathrm{~min}$ each time. Then dehydration was carried out with acetone of different concentrations of 50\%, 60\%,70\%, $80 \%, 90 \%$, and $100 \%$ from low to high for $20 \mathrm{~min}$ each time. The epoxy resin permeated according to a gradient of volume ratio of 3:1, 3:2, 3:3, each for $2 \mathrm{~h}$, and the pure epoxy resin permeated for $24 \mathrm{~h}$. The samples were placed on the embedding plate and added with epoxy resin. The samples were put in an oven at $45^{\circ} \mathrm{C}$ for $12 \mathrm{~h}$. The resin was polymerized, taken out and cooled to room temperature. Sections were cut into $50 \mathrm{~nm}$ thick using a Leica EMUC6 ultramicrotome (Leica Microsystems GmbH, Wentzler, Germany). Then the sections were stained with uranyl acetate and lead citrate for $30 \mathrm{~min}$. The JEM-1400 transmission electron microscope (JEOL Ltd., Tokyo, Japan) was then used to examined and imaged the ultrastructure. The chloroplasts in 10 randomly selected cells were counted the average number of chloroplasts per cell. The length and width of chloroplasts in 10 intact chloroplasts distributed in different cells were measured by AutoCAD.

\subsection{RNA Extraction, cDNA Library Preparation, and Sequencing}

Total RNA was extracted from the colored and green leaves separately using an RNA Purification Kit (Tiangen Biotech Co., Beijing, China) following the manufacturer's instructions. Three replicates for each sample were used. The integrity of the RNA was verified by RNase-free agarose gel electrophoresis and the concentration was measured using a Nano Drop 2000 spectrophotometer (Thermo Scientific, Waltham, MA, USA). High-quality RNA from leaves at each stage was mixed in equal quantities for subsequent RNA sequencing. For each developmental stage of colored and green leaves, three RNA samples were used in the construction of a cDNA library and Illumina sequencing, which was completed by Gene Denovo Biotechnology Co. (Guangzhou, China). The transcriptome data has been uploaded to the NCBI Sequence Read Archive (https://www.ncbi.nlm.nih.gov/sra/) under the accession number SRP238684. 


\subsection{De Novo Assembly of RNA-Seq Reads and Quantifying Gene Expression}

Transcriptome de novo assembly was carried out in this study. Before assembly, adapter sequences were removed from the raw reads. Then, low-quality reads (with over $40 \%$ of bases with quality scores of 10 or lower and/or over $10 \%$ of bases unknown) were removed from each data set to establish more reliable results. Following this, clean, high quality reads from all the samples were combined and assembled using Trinity to construct unique consensus sequences for reference. Sequencing reads were remapped to the reference sequences by SOAP aligner/soap2. For each gene, the expression level was measured by Reads Per Kilobase exon Model per Million mapped reads (RPKM), based on the number of uniquely mapped reads, to eliminate the influence of different gene lengths and sequencing discrepancies in the gene expression calculation. For genes with more than one alternative transcript, the longest transcript was selected to calculate the RPKM.

\subsection{Identification and Functional Analysis of DEGs}

To identify genes that were differentially expressed between the libraries created from colored and green leaves, DEGs from different stages were identified by comparing the expression levels at stage A with those at stage B and stage $C$ in the two leaf types, respectively. To correct for multiple testing, the false discovery rate (FDR) was calculated to adjust the threshold P-value. Transcripts with a fold change $\geq 2$ and FDR $<0.05$ were considered to be differentially expressed between the stages. The DEGs identified were used for Gene Ontology (GO) and Kyoto Encyclopedia of Genes and Genomes (KEGG) enrichment analysis. GO enrichment analysis of all DEGs was implemented using the GO seq $\mathrm{R}$ package, based on Wallenius non-central hypergeometric distribution. GO terms were assigned to the up- and downregulated DEGs, with a corrected $P=0.01$. For pathway enrichment analysis, all DEGs were mapped to pathways in the KEGG database to identify significantly enriched KEGG pathways. DEGs were considered significantly enriched in a metabolic pathway at $q \leq 0.05$ compared with the whole transcriptome background.

\subsection{Validation of DEGs by Quantitative Real-Time PCR (qRT-PCR)}

To validate the results from RNA-Seq and DGE analysis, 22 DEGs associated with chlorophyll metabolism, photosynthesis, biosynthesis of secondary metabolites, and photosynthesis were selected for qRT-PCR. The specific primers used in the experiment to detect the genes expression levels were designed by Primer Premier 5.0 software (Table S1). The OfACT gene was used as an internal control in each qRT-PCR experiment[23]. qRT-PCR was performed using ABI StepOnePlus Systems (Applied Biosystems, Foster city, CA, USA). The RNA samples were quantified using a NanoDrop 2000 spectrophotometer (Thermo Scientific, Waltham, MA, USA) and SYBR Premix Ex Taq (Takara Biotechnology, Dalian, Liaoning Province, China). The cDNAs were synthesized from $5 \mu \mathrm{g}$ total RNA and diluted 10-fold for gene expression experiments. Relative gene expression levels were calculated according to the $2^{-\Delta \Delta C t}$ comparative CT method. The qRT-PCR and DGE analysis results were presented as fold changes in gene expression relative to the control samples.

\section{Results}

\subsection{Phenotypic and Physiological Characterization in Colored and Green Leaves}

The green leaves of $O$. fragrans 'Yinbi Shuanghui' grew on the same plant as colored leaves (Figure 1A). At the earliest stage, the young green leaves appeared dark red, while the young colored leaves were green with a light red edge. The dark red leaves then turned green, while the colored leaves gradually formed a limbate pattern with a yellow-white margin and green middle. Finally, the green area in the middle of the colored leaves gradually expanded and the yellow edge narrowed, but the green leaves remained green (Figure 1B). 


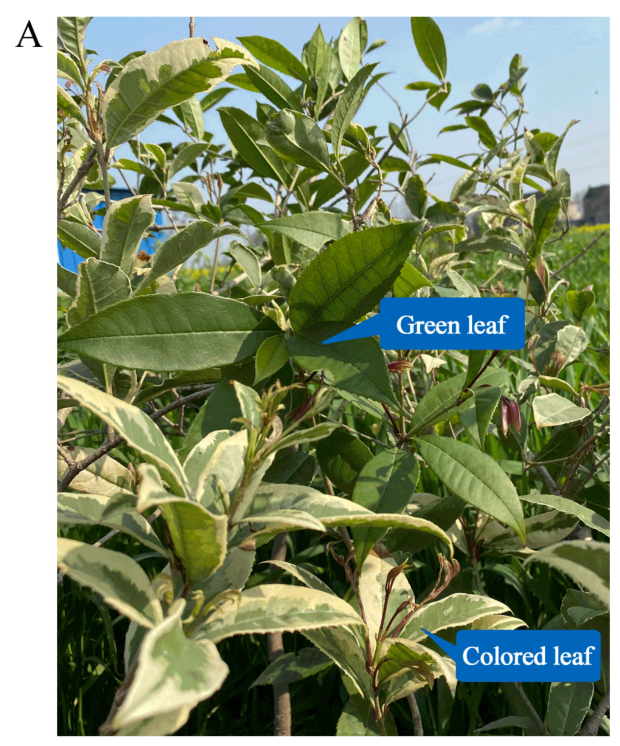

$\mathrm{C}$

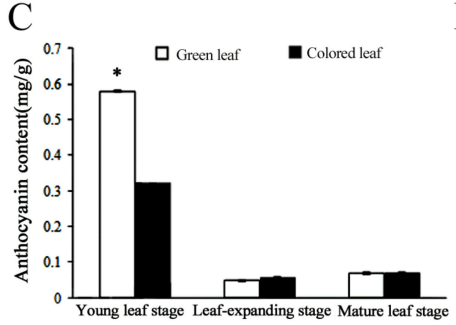

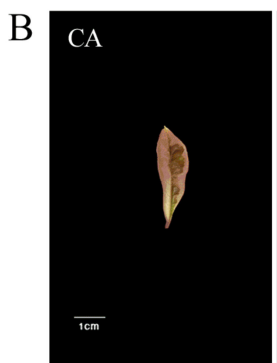

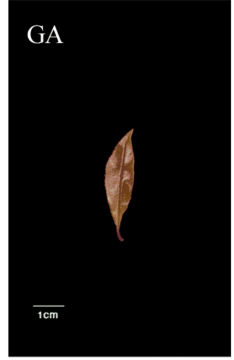

$\mathrm{D}$

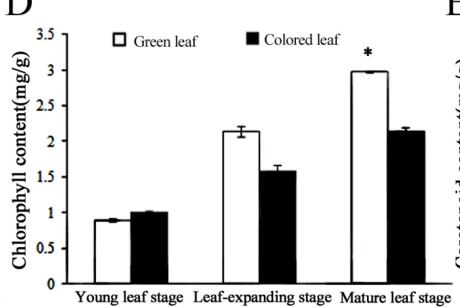

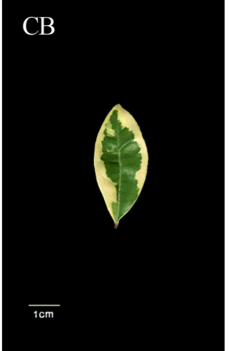
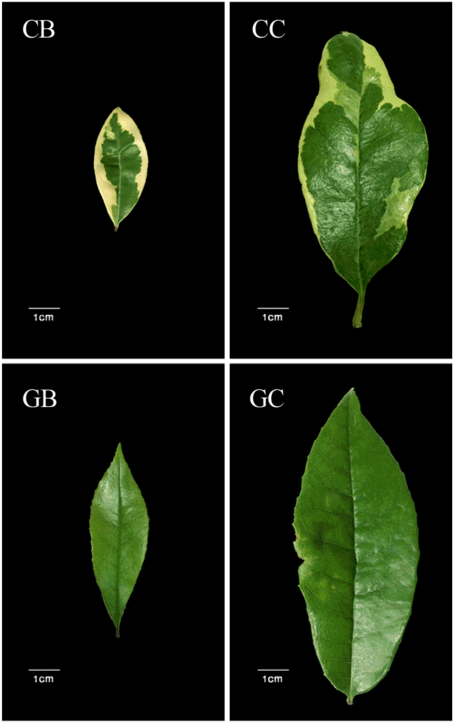

$\mathrm{E}$

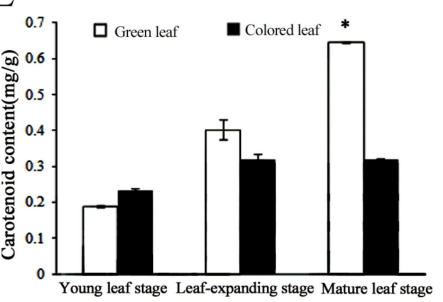

Figure 1. (A) Phenotype of O. fragrans 'Yinbi Shuanghui'; (B) Phenotype of the colored leaf in young leaf stage (CA), leaf-expanding stage (CB), and mature leaf stage (CC); Phenotype of the green leaf in young leaf stage(GA), leaf-expanding stage (GB), and mature leaf stage (GC); (C) Anthocyanin content in colored and green leaf; (D) Chlorophyll content in colored and green leaf; (E) Carotenoid content in colored and green leaf. The significance of differences compared with green leaf is indicated with an asterisk $(p<0.05)$.

To characterize the phenotypic color changes in the leaves, we analyzed the changes in pigment content in the green and colored leaves. The anthocyanin content of green leaves was significantly higher than that of colored leaves at the young leaf stage (Figure 1C). The total chlorophyll and carotenoid contents of the two leaf types increased during leaf development. The content of chlorophyll and carotenoid in green leaves was significantly higher than that in colored leaves at the mature leaf stage (Figure 1D,E). Chl a/Chl b showed significant differences at the young leaf stage and mature leaf stage (Table 1$)$.

Table 1. Comparison of pigment content in colored and green leaf.

\begin{tabular}{|c|c|c|c|c|c|}
\hline Sample & $\begin{array}{l}\text { Chlorophyll } \\
\left(\mathrm{mg} \cdot \mathrm{g}^{-1}\right)\end{array}$ & $\begin{array}{l}\text { Chlorophyll a } \\
\quad\left(\mathrm{mg}^{-1} \mathrm{~g}^{-1}\right)\end{array}$ & $\begin{array}{l}\text { Chlorophyll b } \\
\quad\left(\mathrm{mg} \cdot \mathrm{g}^{-1}\right)\end{array}$ & $\begin{array}{l}\text { Carotenoid } \\
\left(\mathrm{mg} \cdot \mathrm{g}^{-1}\right)\end{array}$ & Chl a/Chl b \\
\hline GA & $0.89 \pm 0.02 \mathrm{~d}$ & $0.41 \pm 0.00 \mathrm{~d}$ & $0.47 \pm 0.02 \mathrm{~d}$ & $0.19 \pm 0.00 \mathrm{~d}$ & $0.88 \pm 0.03 d$ \\
\hline GB & $2.13 \pm 0.07 b$ & $1.21 \pm 0.07 b$ & $0.92 \pm 0.03 b$ & $0.40 \pm 0.03 b$ & $1.31 \pm 0.08 b$ \\
\hline GC & $2.97 \pm 0.01 \mathrm{a}$ & $1.88 \pm 0.03 a$ & $1.09 \pm 0.03 a$ & $0.65 \pm 0.00 \mathrm{a}$ & $1.73 \pm 0.07 a$ \\
\hline CA & $1.00 \pm 0.01 \mathrm{~d}$ & $0.50 \pm 0.01 d$ & $0.50 \pm 0.02 \mathrm{~d}$ & $0.23 \pm 0.01 \mathrm{~d}$ & $1.01 \pm 0.05 c$ \\
\hline $\mathrm{CB}$ & $1.58 \pm 0.08 c$ & $0.88 \pm 0.05 c$ & $0.70 \pm 0.03 c$ & $0.31 \pm 0.02 c$ & $1.26 \pm 0.02 b c$ \\
\hline $\mathrm{CC}$ & $2.14 \pm 0.05 b$ & $1.23 \pm 0.01 b$ & $0.91 \pm 0.05 b$ & $0.32 \pm 0.00 c$ & $1.35 \pm 0.07 b$ \\
\hline
\end{tabular}

The colored leaf in young leaf stage (CA), leaf-expanding stage (CB), and mature leaf stage (CC); the green leaf in young leaf stage (GA), leaf-expanding stage (GB), and mature leaf stage (GC); Different letters denote significant differences according to Tukey test at the 0.05 level. 
We further compared the ultrastructure of the chloroplasts in green leaves and the green and yellow parts of colored leaves. In the mesophyll cells of the green leaves (Figure $2 \mathrm{~A}(\mathrm{a}-\mathrm{c})$ ), chloroplasts developed typical structures, with a typical granum thylakoid structure consisting of thylakoids, numerous starch grains, and few plastoglobuli. There were few chloroplasts in the green parts of the colored leaves and their development was seen to be abnormal (Figure $2 \mathrm{~A}(\mathrm{~d}-\mathrm{f})$ ). There was no obvious membrane structure and a small number of granum thylakoids and plastoglobuli. In the yellow part of the colored leaf (Figure 2A(g-i)), there were more stromal lamellae and the thylakoids were not normal. The number of granum thylakoids was low, but there was a large number of plastoglobuli. The number of chloroplasts in green leaves was significantly higher than that in colored leaves. There were significant differences in chloroplast length and width between green leaves and yellow parts of colored leaves at the mature leaf stage (Figure $2 \mathrm{C}-\mathrm{E}$ ). The results indicated that the changes in chloroplast structure in the colored leaves affected the pigment distribution.

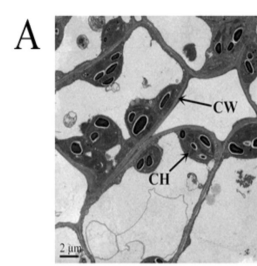

a

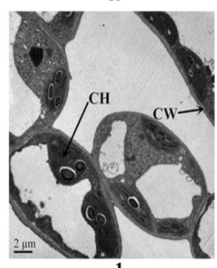

d

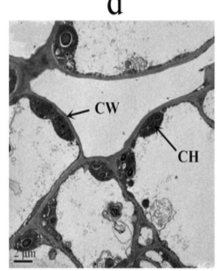

g

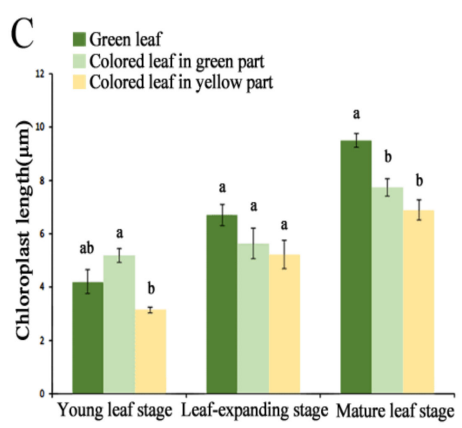

b

e
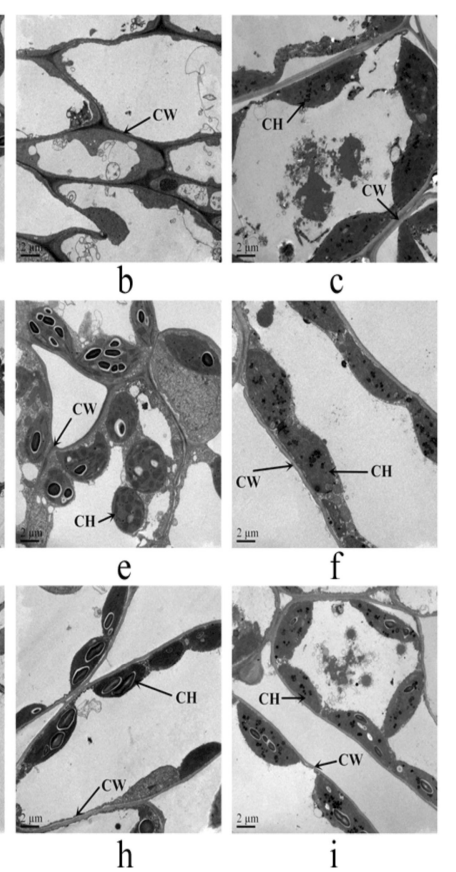

D Green leaf

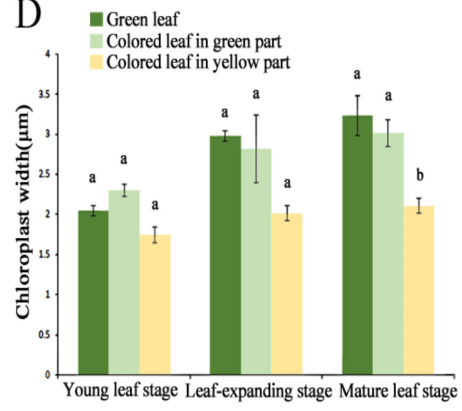

C

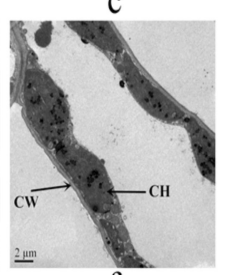

m

p
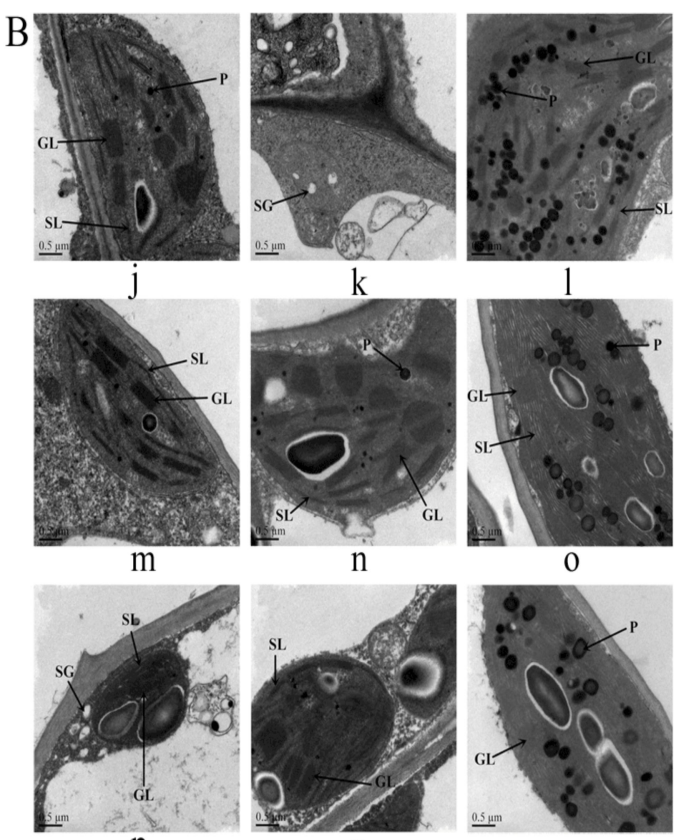

n
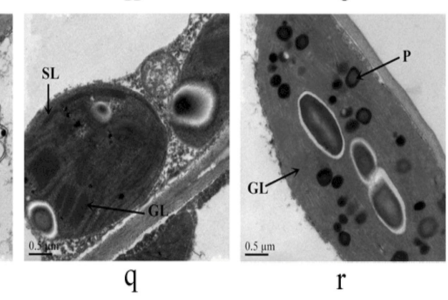

$\begin{aligned} \text { E } & \text { Green leaf } \\ & \text { Colored leaf in green part } \\ & \text { Colored leaf in yellow part }\end{aligned}$

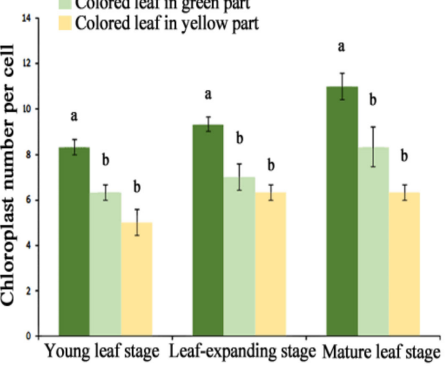

Figure 2. Comparison of ultrastructure of chloroplasts in colored and green leaf. (A) Bar $=2 \mu \mathrm{m}(\mathbf{a}-\mathbf{i})$; (B) Bar $=0.5 \mu \mathrm{m}(\mathbf{j}-\mathbf{r}) ;$ (C) Chloroplasts length in colored and green leaf; (D) Chloroplasts width in colored and green leaf; (E) Chloroplasts number per cell in colored and green leaf. Different letters in the same stage denote significant differences according to Tukey test at the 0.05 level. Chloroplasts ultrastructure of green leaf from young leaf stage to mature leaf stage $(\mathbf{a}-\mathbf{c}, \mathbf{j}-\mathbf{1})$; Chloroplasts ultrastructure of colored leaf in green part from young leaf stage to mature leaf stage $(\mathbf{d}-\mathbf{f}, \mathbf{m}-\mathbf{o})$; Chloroplasts ultrastructure of colored leaf in yellow part from young leaf stage to mature leaf stage (g-i,p-r); $\mathrm{CH}$ : chloroplasts; $\mathrm{CW}$ : cell wall; GL: granum thylakoid; SL: stromal lamellae; SG: starch grain; P: plastoglobuli. 


\subsection{Transcriptome Analysis}

A total of 18 samples of colored and green leaves from different developmental stages of $O$. fragrans 'Yinbi Shuanghui' were sequenced. After removing the low mass and linker sequences, 18 samples provided $755,544,800$ clean reads with a total of $117,890,827,944$ bases. The values for each sample were shown in Table 2. The percentage of Q20 was 95\% or more. The GC content of each sample ranged from $47.43-45.75 \%$. The ratio of clean reads from each sample ranged from $94.01-97.79 \%$. The mapping ratio, which was compared with the reference sequence, ranged from $88.46-86.11 \%$ (Table 3 ). The concentration of the three biological repeats was relatively concentrated, indicating that there was good reproducibility between the repeats and sequencing results (Figure S1).

Table 2. Summary of sequencing data.

\begin{tabular}{|c|c|c|c|c|c|c|}
\hline Sample & Raw Read & Clean Read & GC\% & Base Pair & Q20 (\%) & Q30 (\%) \\
\hline CA-1 & 50136154 & $\begin{array}{l}48697992 \\
(97.13 \%)\end{array}$ & $45.75 \%$ & 7123264976 & $\begin{array}{c}6965309247 \\
(97.78 \%)\end{array}$ & $\begin{array}{c}6650946216 \\
(93.37 \%)\end{array}$ \\
\hline CA-2 & 47247834 & $\begin{array}{l}45806568 \\
(96.95 \%)\end{array}$ & $47.08 \%$ & 6692400673 & $\begin{array}{c}6537141040 \\
(97.68 \%)\end{array}$ & $\begin{array}{c}6229471306 \\
(93.08 \%)\end{array}$ \\
\hline CA-3 & 43851748 & $\begin{array}{c}42713330 \\
(97.4 \%)\end{array}$ & $46.62 \%$ & 6255173639 & $\begin{array}{c}6122879028 \\
(97.89 \%)\end{array}$ & $\begin{array}{c}5853739955 \\
(93.58 \%)\end{array}$ \\
\hline CB-1 & 41291246 & $\begin{array}{l}40007934 \\
(96.89 \%)\end{array}$ & $46.41 \%$ & 5851697788 & $\begin{array}{c}5719672342 \\
(97.74 \%)\end{array}$ & $\begin{array}{c}5456822949 \\
(93.25 \%)\end{array}$ \\
\hline CB-2 & 39691580 & $\begin{array}{l}38508004 \\
(97.02 \%)\end{array}$ & $46.49 \%$ & 5630898128 & $\begin{array}{c}5499410453 \\
(97.66 \%)\end{array}$ & $\begin{array}{c}5240305903 \\
(93.06 \%)\end{array}$ \\
\hline CB-3 & 41987310 & $\begin{array}{l}40869684 \\
(97.34 \%)\end{array}$ & $46.78 \%$ & 5982309095 & $\begin{array}{c}5851983440 \\
(97.82 \%)\end{array}$ & $\begin{array}{c}5589280458 \\
(93.43 \%)\end{array}$ \\
\hline CC-1 & 40240238 & $\begin{array}{l}38318272 \\
(95.22 \%)\end{array}$ & $47.11 \%$ & 5496433178 & $\begin{array}{c}5292402611 \\
(96.29 \%)\end{array}$ & $\begin{array}{c}4930302996 \\
(89.70 \%)\end{array}$ \\
\hline CC-2 & 47464442 & $\begin{array}{l}45485024 \\
(95.83 \%)\end{array}$ & $46.30 \%$ & 6547342914 & $\begin{array}{c}6313888832 \\
(96.43 \%)\end{array}$ & $\begin{array}{c}5896493760 \\
(90.06 \%)\end{array}$ \\
\hline CC-3 & 45207378 & $\begin{array}{l}43368058 \\
(95.93 \%)\end{array}$ & $46.12 \%$ & 6246637041 & $\begin{array}{c}6025421211 \\
(96.46 \%)\end{array}$ & $\begin{array}{c}5629644889 \\
(90.12 \%)\end{array}$ \\
\hline GA-1 & 47923416 & $\begin{array}{l}46863062 \\
(97.79 \%)\end{array}$ & $46.51 \%$ & 6920028257 & $\begin{array}{c}6813658441 \\
(98.46 \%)\end{array}$ & $\begin{array}{c}6592998268 \\
(95.27 \%)\end{array}$ \\
\hline GA-2 & 52077614 & $\begin{array}{l}49301610 \\
(94.67 \%)\end{array}$ & $47.43 \%$ & 7040828776 & $\begin{array}{c}6764478452 \\
(96.08 \%)\end{array}$ & $\begin{array}{c}6283883279 \\
(89.25 \%)\end{array}$ \\
\hline GA-3 & 47559986 & $\begin{array}{l}45334370 \\
(95.32 \%)\end{array}$ & $47.04 \%$ & 6503246887 & $\begin{array}{c}6258755974 \\
(96.24 \%)\end{array}$ & $\begin{array}{c}5827855536 \\
(89.61 \%)\end{array}$ \\
\hline GB-1 & 47687778 & $\begin{array}{l}45908678 \\
(96.27 \%)\end{array}$ & $46.64 \%$ & 6616481347 & $\begin{array}{c}6389425806 \\
(96.57 \%)\end{array}$ & $\begin{array}{c}5978986991 \\
(90.37 \%)\end{array}$ \\
\hline GB-2 & 40856522 & $\begin{array}{l}38407958 \\
(94.01 \%)\end{array}$ & $46.54 \%$ & 5441768736 & $\begin{array}{c}5187774775 \\
(95.33 \%)\end{array}$ & $\begin{array}{c}4761428460 \\
(87.50 \%)\end{array}$ \\
\hline GB-3 & 50998640 & $\begin{array}{l}49742274 \\
(97.54 \%)\end{array}$ & $46.50 \%$ & 7337535698 & $\begin{array}{c}7216493845 \\
(98.35 \%)\end{array}$ & $\begin{array}{c}6971524292 \\
(95.01 \%)\end{array}$ \\
\hline GC-1 & 45633624 & $\begin{array}{l}44576264 \\
(97.68 \%)\end{array}$ & $46.68 \%$ & 6583222002 & $\begin{array}{c}6478966906 \\
(98.42 \%)\end{array}$ & $\begin{array}{c}6263761501 \\
(95.15 \%)\end{array}$ \\
\hline GC-2 & 52890664 & $\begin{array}{l}51635718 \\
(97.63 \%)\end{array}$ & $47.04 \%$ & 7626415754 & $\begin{array}{c}7502551431 \\
(98.38 \%)\end{array}$ & $\begin{array}{c}7248121801 \\
(95.04 \%)\end{array}$ \\
\hline GC-3 & 55658706 & $\begin{array}{l}54186622 \\
(97.36 \%)\end{array}$ & $46.75 \%$ & 7995143055 & $\begin{array}{c}7859478736 \\
(98.30 \%)\end{array}$ & $\begin{array}{c}7585090670 \\
(94.87 \%)\end{array}$ \\
\hline
\end{tabular}

The colored leaf in young leaf stage (CA), leaf-expanding stage (CB), and mature leaf stage (CC); the green leaf in young leaf stage (GA), leaf-expanding stage (GB), and mature leaf stage (GC);1-3: Three biological replicates of each leaf sample; Raw reads: original number of reads obtained by sequencing; Clean reads: number of reads after removing low-quality reads and trimming adapter sequences; GC\%: percentage of $\mathrm{G}$ and $\mathrm{C}$ in total bases; Base pair: number of DNA base pair; Q20: Phred score, indicates 99\% accuracy of sequenced bases; Q30: Phred score, indicates $99.9 \%$ accuracy of sequenced bases. 
Table 3. High quality clean reads mapped to the reference sequence.

\begin{tabular}{ccccc}
\hline Sample & All Reads Num & $\begin{array}{c}\text { UniqueMapped } \\
\text { Reads }\end{array}$ & $\begin{array}{c}\text { Multiple Mapped } \\
\text { Reads }\end{array}$ & Mapping Ratio \\
\hline CA-1 & 47837392 & 35574913 & 5619933 & $86.11 \%$ \\
CA-2 & 45460760 & 34139028 & 5403742 & $86.98 \%$ \\
CA-3 & 41853268 & 31431109 & 4984757 & $87.01 \%$ \\
CB-1 & 39921676 & 30448781 & 4373979 & $87.23 \%$ \\
CB-2 & 38064446 & 29044117 & 4312621 & $87.63 \%$ \\
CB-3 & 40736136 & 31124068 & 4696715 & $87.93 \%$ \\
CC-1 & 38049842 & 29707713 & 3795059 & $88.05 \%$ \\
CC-2 & 45215906 & 35061304 & 4497542 & $87.49 \%$ \\
CC-3 & 43259684 & 33546908 & 4150829 & $87.14 \%$ \\
GA-1 & 46725254 & 35483751 & 4884461 & $86.39 \%$ \\
GA-2 & 48854482 & 36860201 & 5709217 & $87.14 \%$ \\
GA-3 & 45201006 & 34009232 & 5270798 & $86.90 \%$ \\
GB-1 & 45666432 & 34822812 & 5256176 & $87.76 \%$ \\
GB-2 & 38305776 & 28947778 & 4418419 & $87.10 \%$ \\
GB-3 & 49619384 & 37946864 & 5267936 & $87.09 \%$ \\
GC-1 & 44405898 & 35133689 & 4016541 & $88.16 \%$ \\
GC-2 & 51159608 & 40525142 & 4731774 & $88.46 \%$ \\
GC-3 & 53974230 & 42462991 & 4936445 & $87.82 \%$ \\
\hline
\end{tabular}

The colored leaf in young leaf stage (CA), leaf-expanding stage (CB), and mature leaf stage (CC); the green leaf in young leaf stage (GA), leaf-expanding stage (GB), and mature leaf stage (GC);1-3: Three biological replicates of each leaf sample; All Reads Num: total number of reads after ribosome removal; Unique Mapped Reads: number of reads on unique alignment with reference sequence; Multiple Mapped Reads: number of reads on multiple alignments with reference sequence; Mapping Ratio = (Unique Mapped Reads + Multiple Mapped Reads)/All Reads Num.

\subsection{Identification and Functional Annotation of DEGs during Leaf Development}

To identify variations in gene expression between the colored and green leaves at different stages, we used the RPKM values to calculate the DEGs. The DEGs were identified and filtered using the following criteria: $p<0.05$ and $\log _{2}$ (fold change) $>1$. We compared the DEGs in the colored and green leaves during leaf development (Figure 3). The volcano plots map was constructed with DEGs to show the expression patterns of all the samples at different stages (Figure S2). In the young leaf stage, there were 11,881 unique DEGs in the two leaf types and the DEGs were mainly upregulated: 6852 were upregulated and 5029 were downregulated. In the leaf-expanding stage, there were 696 DEGs. Among these, there were 314 upregulated and 382 downregulated genes. At the mature leaf stage, there were 785 DEGs, of which 211 were upregulated and 574 were downregulated.

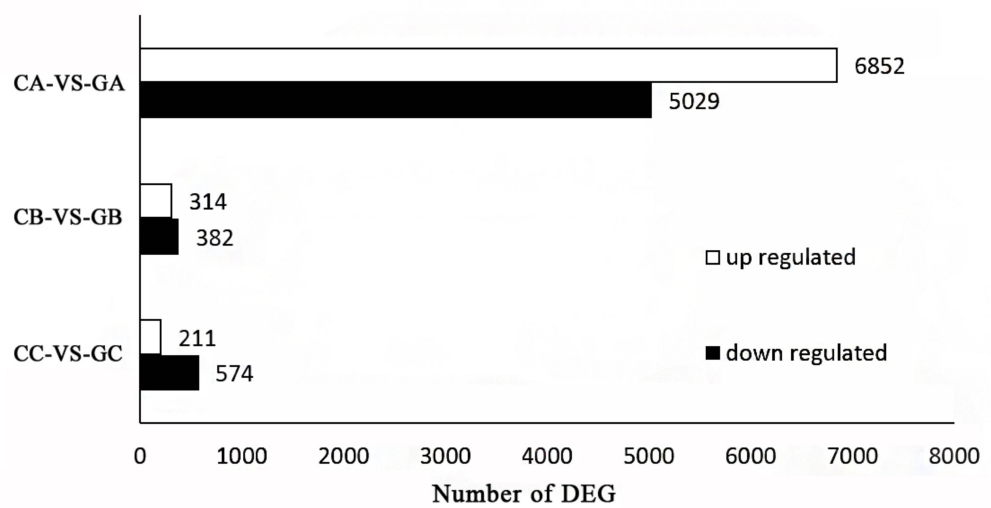

Figure 3. DEGs in colored and green leaves. The colored leaf in young leaf stage (CA), leaf-expanding stage (CB), and mature leaf stage (CC); the green leaf in young leaf stage (GA), leaf-expanding stage (GB), and mature leaf stage (GC). 
The functions of these DEGs were classified according to the GO database using the Blast2GO software suite. GO analysis found that DEGs in colored and green leaves were mainly concentrated in 'molecular function', 'cell composition', and 'biological processes'. For the colored and green leaves in the young leaf stage (Figure 4A), the GO term of the upregulated DEGs was significantly enriched in cellular processes $(1495,21.82 \%)$, metabolic processes $(1464,21.37 \%)$, and binding $(1448,21.13)$. Downregulated DEGs were significantly enriched in binding $(1448,21.13 \%)$, catalytic activity $(1417$, $20.68 \%$ ), and metabolic processes (1151, 22.89\%). In the leaf-expanding stage (Figure 4B), the GO terms for the upregulated DEGs in colored and green leaves were significantly enriched in metabolic processes $(75,23.89 \%)$, binding $(65,20.70 \%)$, and cellular processes $(59,18.79 \%)$, whereas the downregulated DEGs were significantly enriched in metabolic processes $(92,24.08 \%)$, catalytic activity $(72,18.85 \%)$, and cellular processes $(66,17.28 \%$ ). During the mature leaf stage (Figure $4 \mathrm{C})$, the GO term of the upregulated DEGs was significantly enriched in catalytic activity $(51,24.17 \%)$, metabolic processes (46, $21.80 \%)$, and binding $(41,19.43 \%)$. However, the downregulated DEGs were significantly enriched in catalytic activity $(132,23.00 \%)$, metabolic processes $(128,22.30 \%)$, cellular processes $(120,20.91 \%)$, and combinations $(120,20.91 \%)$.

There were significant differences in the KEGG metabolic pathways enriched by DEGs in colored and green leaves at different stages. In the young leaf stage, there were 1915 DEGs with pathway annotation in colored and green leaves. The highest expressed DEG was the gene encoding the ribosomal protein (Table S2). These DEGs were enriched in 127 KEGG pathways and the number of DEGs enriched in each pathway was different (Figure 4D). Six of the pathways were significantly enriched (Table S3). DNA replication was the most significant enrichment pathway, followed by the pyrimidine metabolic pathway, anthocyanin biosynthetic pathway, RNA polymerase pathway, and flavonoid biosynthesis pathway. Among these, the upregulated genes in colored leaves associated with leaf color expression were significantly enriched in the anthocyanin biosynthesis and flavonoid biosynthesis pathways, with 14 and 18 DEGs, respectively. Significant downregulation of genes in colored leaves was seen in the photosynthesis pathway.

During the leaf-expanding stage, we performed a pathway analysis of 147 DEGs in colored and green leaves. The highest expressed DEGs were the genes encoding ribosomal protein and cytochrome c oxidase (Table S2). Ribosomal protein played an important role in the translation of key proteins involved in chloroplast development and photosynthesis [24]. A decrease in cytochrome c oxidase activity produced strong alterations of plant growth and development [25]. Our study found that the genes encoding ribosomal protein and cytochrome c oxidase were downregulated in colored leaves. The downregulated expression of these genes may be responsible for the change in leaf color. As shown, the DEGs were enriched in 72 KEGG pathways (Figure 4E). Four of the pathways were significantly enriched (Table S4). These were the protein processing pathway in the endoplasmic reticulum, flavonoid biosynthesis pathway, organic selenium-containing compound metabolic pathway, and monoterpenoid biosynthetic pathway. In colored leaves, the upregulated genes associated with leaf color expression were significantly enriched in anthocyanin biosynthesis and flavonoid biosynthesis, whereas the pathway in which the downregulated genes were significantly enriched was that for phenylpropanoid biosynthesis.

There were 141 DEGs in the colored and green leaves at the mature leaf stage, which were enriched in 76 KEGG pathways (Figure 4F). The highest expressed DEGs were the genes encoding crystal protein and camp-regulated $\mathrm{d} 2$ protein (Table S2). Only the biosynthesis of amino acid pathway was significantly enriched (Table S5). However, there was no significantly enriched pathways associated with leaf color expression. 

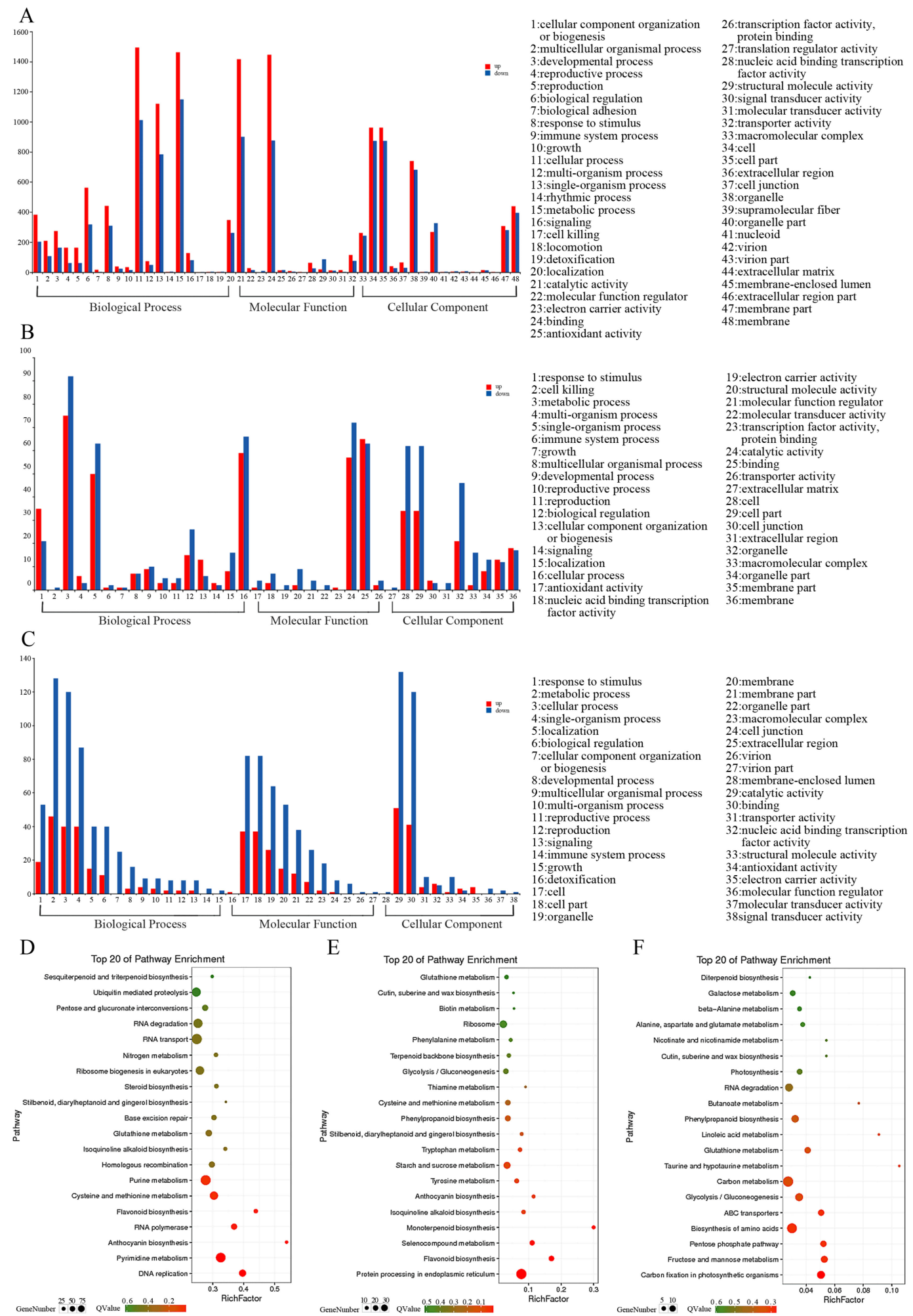

Figure 4. Gene Ontology (GO) classification of unigenes and Top 20 enriched KEGG pathway among the annotated DEGs of colored and green leaf in different leaf stages. (A,D) CA-VS-GA; (B,E) CB-VS-GB; $(\mathbf{C}, \mathbf{F})$ CC-VS-GC. The colored leaf in young leaf stage (CA), leaf-expanding stage (CB), and mature leaf stage (CC); the green leaf in young leaf stage (GA), leaf-expanding stage (GB), and mature leaf stage (GC). 


\subsection{Identification of DEGs Related to Pigment Metabolism}

\subsubsection{Identification of DEGs Related to Chlorophyll Metabolism}

Among all the annotated pathways, we focused on pathways closely related to pigment synthesis, such as the chlorophyll, carotenoid, and secondary metabolic pathways. To better understand the chlorophyll metabolic in green and colored leaves, the enzymes involved in the porphyrin and chlorophyll metabolism pathways were investigated, and the expression of genes encoding these enzymes was significantly different. (Figure 5A). We found that there were DEGs with significant differences in the chlorophyll metabolism pathway at the young leaf stage and the differences in DEGs between the leaf-expanding and mature leaf stages were not significant. During the young leaf stage, 16 genes were found to annotate 11 enzymes in the porphyrin and chlorophyll metabolic pathways. There were 11 DEGs involved in chlorophyll synthesis. Unigene0010257, encoding Glutamyl t-RNA reductase (HEMA1), was significantly upregulated during the young leaf stage in green leaves and its expression was generally upregulated during all three stages. There were also five DEGs involved in chlorophyll degradation, including Unigene0028398 and Unigene0028399, encoding heme oxygennase-1 (HO1), Unigene0029892, encoding pheophorbide a oxidase (PAO), Unigene0017904, encoding pheophytin hydrolase $(P P H)$, and Unigene0010601, encoding chlorophyllase-2 (CLH2). They were significantly downregulated in green leaves compared with those in colored leaves at the young leaf stage. PAO and CLH2 were key regulatory enzymes in the chlorophyll degradation pathway. The results indicated that the downregulated expression of the coding genes was associated with lower chlorophyll content in the colored leaves.

Overexpression of the SGR can promote the decomposition of chlorophyll and reduce the number of granum thylakoids and chlorophyll content in normally-growing leaves. In this study, we identified seven DEGs associated with the SGR, based on KEGG pathway enrichment analysis (Figure 5B). Six $S G R$ were significantly upregulated in colored leaves during the young leaf stage. At the mature leaf stage, Unigene0050901 encoding SGR, showed the same trend and was significantly upregulated in the colored leaves compared with those in green leaves.

Plant photosynthesis was mainly carried out in chloroplasts. The coloration mechanism of plant leaves was closely related to the development and distribution of chloroplasts. In our study, Unigene0039679, which encoded the GLK transcription factor associated with chloroplast development, was downregulated in the three stages of colored leaves and significantly downregulated compared with those in green leaves at the young leaf stage (Figure 5C). This indicated that the chloroplast structure of the colored leaves was different from that of the green leaves in the early stage of leaf development.

\subsubsection{Identification of DEGs Related to Carotenoid Metabolism}

In the young leaf stage, we determined that 16 DEGs with significant differences in the carotenoid pathway. Unigene0036601, encoding phytoene synthase 1 (PSY1), and Unigene0044025, encoding 1-Deoxyxylulose-5-phosphate synthase (DXS), were related to carotenoid synthesis, were significantly downregulated in colored leaves (Figure 5D). Among them, Unigene0044025 was significantly downregulated during the three developmental stages of colored leaves. However, Unigene0033055, encoding 9-cis-epoxycarotenoid dioxygenase (NCED), related to carotene degradation, was significantly upregulated in colored leaves. These results indicated that the expression level of the genes related to carotenoid synthesis in colored leaves was lower than that in green leaves. 
A Porphyrin and chlorophyll metabolism

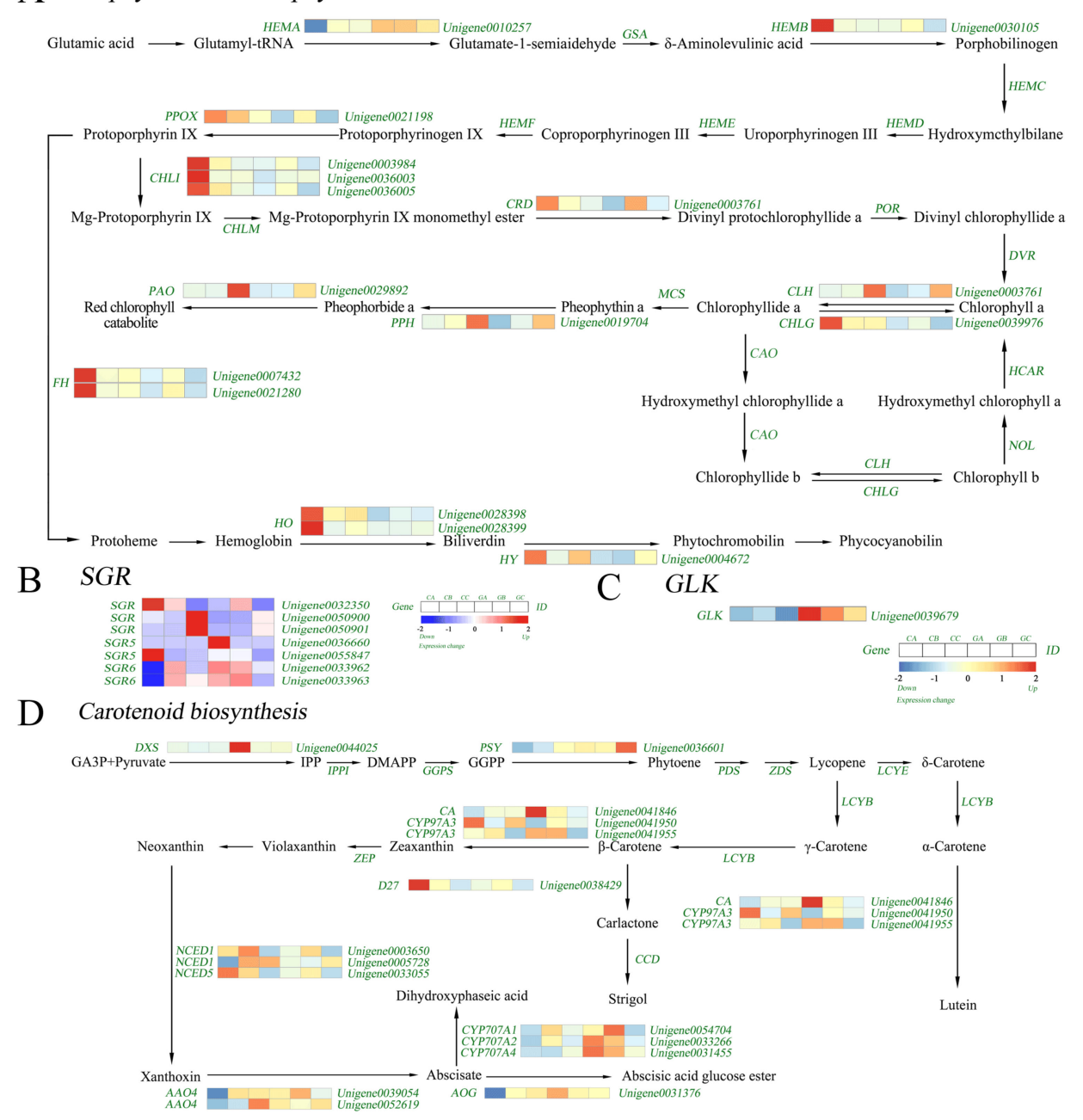

E Secondary metabolism

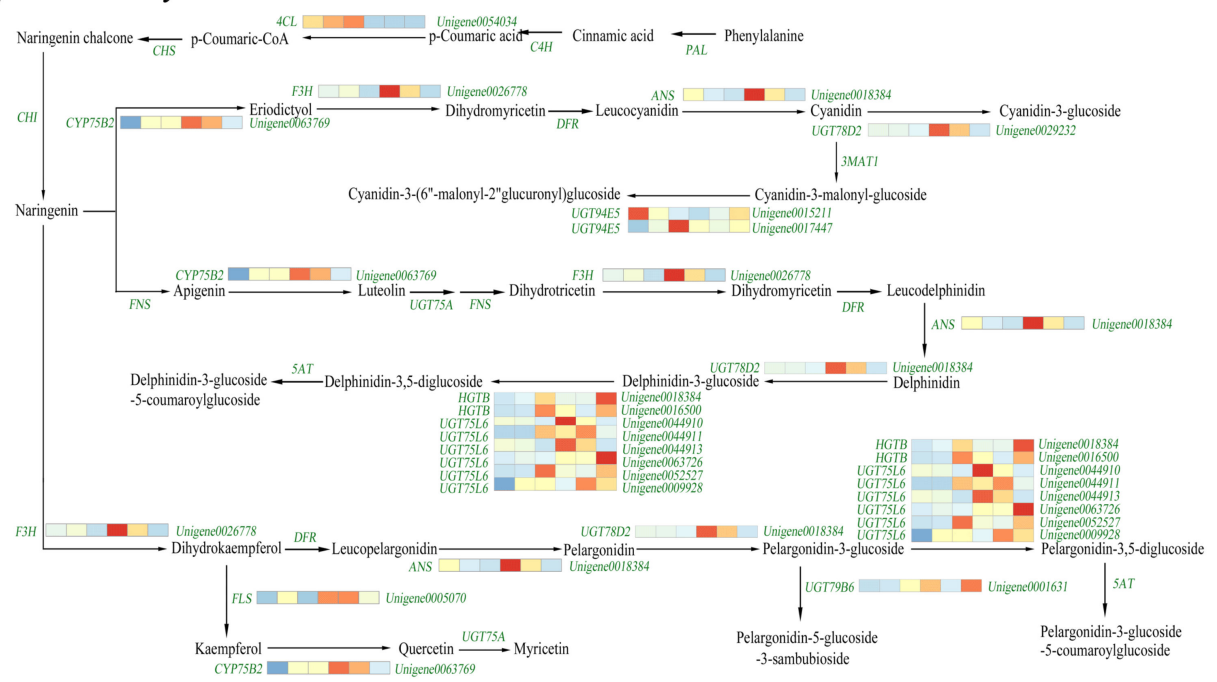

Figure 5. (A) Expression profiles of differentially expressed genes (DEGs) involved in porphyrin and chlorophyll metabolism between colored and green leaf; (B) Expression profiles of DEGs involved in SGR between colored and green leaf; (C) Expression profiles of DEG involved in GLK between colored and green leaf; (D) Expression profiles of DEGs involved in carotenoid biosynthesis between colored and green leaf; (E) Expression profiles of DEGs involved in biosynthesis of secondary metabolites between colored and green leaf. 


\subsubsection{Identification of DEGs Related to Secondary Metabolism}

In this study, 6 DEGs related to the flavonoid synthesis pathway were found (Figure 5E). In the young leaf stage, the expression of Unigene0026778 encoding flavanone 3-hydroxylase $(F 3 H)$, and Unigene0018384 encoding anthocyanidin synthase (ANS) were significantly lower in the colored leaves than in the green leaves. Further analysis of the anthocyanin biosynthesis pathway revealed 14 DEGs. Six genes encoding UDP-glucosyltransferase (UGT75L6) were significantly different in the mature leaf stage of green leaves and colored leaves. The expression of Unigene0015211 encoding UDP-glucosyltransferase (UGT94E5) was higher in the colored leaves of the three stages than in the green leaves. The above results indicated that the upregulated expression of genes involved in the secondary metabolic pathway might be related to the formation of colored leaves at the young leaf stage.

\subsection{Identification of DEGs Co-Expression Modules by WGCNA}

RNA-seq sequencing was used to analyze the DEGs of colored and green leaves. At the same time, WGCNA was used to analyze the association between the co-expressed gene modules formed by DEGs and physiological indicators. Studies had found that the photosynthetic pigment content of colored leaf plant can affect photosynthetic performance [26]. In this study, the pigment content and parameters of photosynthesis and fluorescence in green and colored leaves were measured as physiological indicators. Low levels of gene expression were filtered out by data analysis to obtain 39,965 highly expressed genes. The gene clustering tree was constructed according to the correlation between the expression levels of genes and the gene module. The modules were divided according to the clustering relationship between genes. The modules with similar expression patterns were then merged according to the similarity of the module feature values (Figure S3). By screening the weight values, a total of 15 co-expression modules were obtained. The lavenderblush1 module contained the largest number of genes, 9213 genes, and the Navajo white module contained the lowest number of genes, 88 genes.

By calculating the correlation between the eigenvalues and traits of each module, we found that the ME display of the orange module and the lavenderblush1 module was highly correlated with the physiological indicators of leaf coloration. Chlorophyll and carotenoid metabolism were positively correlated with the lavenderblush1 module and negatively correlated with the orange module (Figure 6). This indicated that the lavenderblush1 module might play an important role in colored leaves, whereas the orange module may play an inhibitory role. In analyzing the enrichment of chlorophyll metabolism- and carotenoid metabolism-related genes, we found that the coral module contained the largest number of DEGs involved in chlorophyll metabolism, but only one DEG related to carotenoid metabolism. The lavenderblush1 module contained 14 DEGs related to the chlorophyll synthesis pathway and 14 DEGs associated with carotenoid metabolism (Table 4). We speculate that the lavenderblush1 module may be related to the color formation mechanism.

To systematically study the regulation of chlorophyll and carotenoid metabolism, we extracted transcription factors from the lavenderblush1 module. The lavenderblush1 module had 261 transcription factors belonging to 52 transcription factor families (Table S6). The main transcription factor families were WRKY (41 unigenes), bHLH (30 unigenes), and ERF (26 unigenes) (Figure S4). Figure 7 showed that these transcription factors exhibited different expression patterns. Cluster analysis among three biological repeats showed that the expression patterns of transcription factors in green and colored leaves were different during the development stage (Figure S5). 


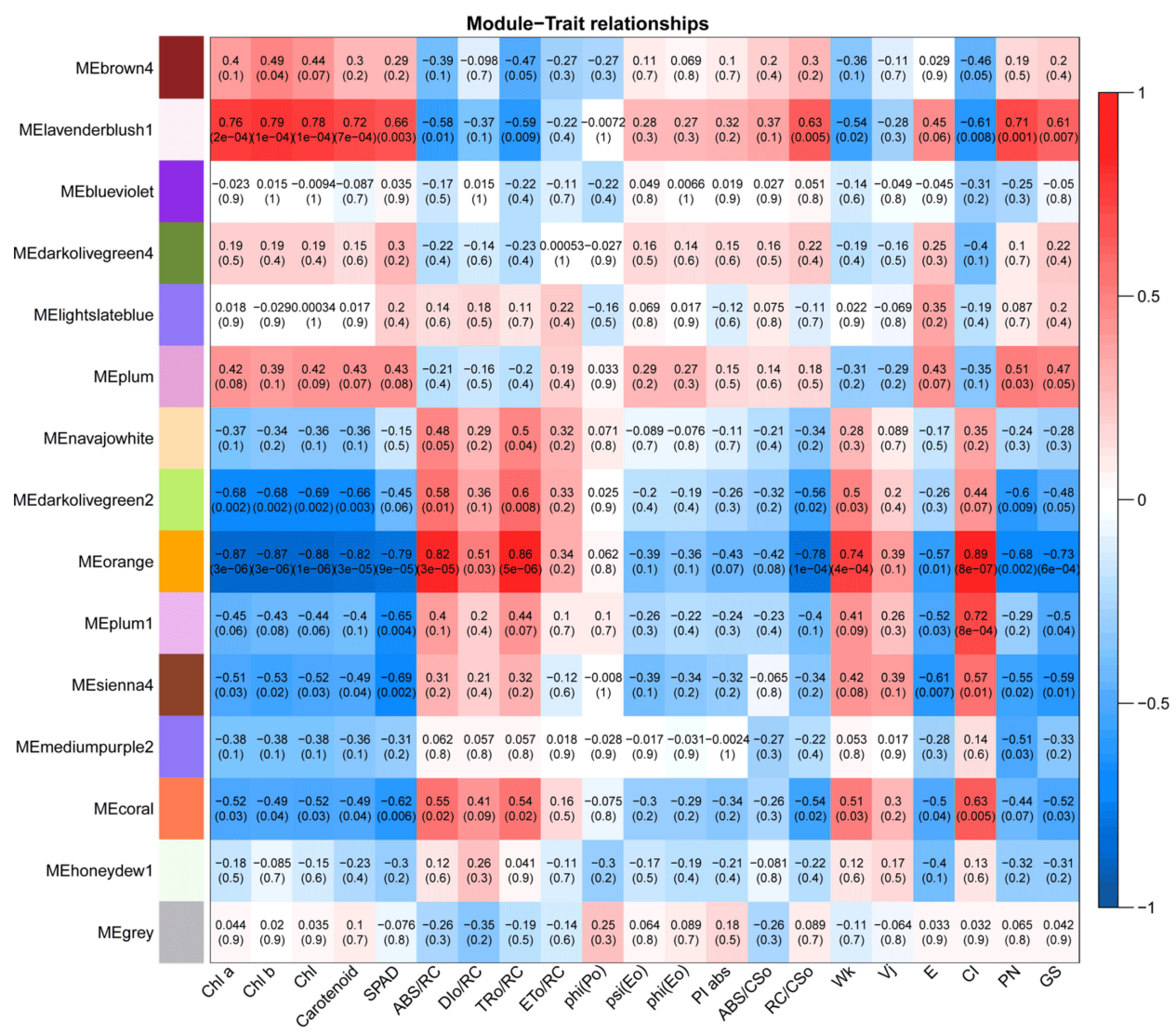

Figure 6. Trait-module associated heat map. Each column represents a physiological indicator, and each row represents a genetic module. The number in each grid represents the correlation between the module and the trait. The number in parentheses represents the significance P-value. The smaller the P-value, the stronger the significance of the representativeness and module correlation. Chl a: content of chlorophyll a; Chl b: content of chlorophyll b; Chl: content of chlorophyll; Carotenoid: content of carotenoid; SPAD: chlorophyll relative value; ABS/RC: photon absorbance rate per active reaction center; $\mathrm{DIo} / \mathrm{RC}$ : exciton dissipation rate per active reaction center; TRo/RC: exciton trapping rate per active reaction center; ETo/RC: electron transport per active reaction center; phi(Po): maximum efficiency of PSII photochemistry; psi(Eo): the efficiency with which a trapped exciton can move an electron into electron transport chain further than QA; phi(Eo): the quantum yield of electron transport; PI abs: performance index on absorption basis.; ABS/CSo: photon absorbance per excited cross-section; RC/CSo: number of reaction centers per excited cross-section; Wk: the ratio of variable fluorescence Fk to amplitude Fo-Fj; Vj: the ratio of variable fluorescence Fj to amplitude Fo-Fp; E: transpiration rate; CI: intercellular; $\mathrm{CO}_{2}$; PN: photosynthetic rate; GS: stomatal conductivity.

Table 4. Distribution of genes associated with leaf color formation in modules.

\begin{tabular}{ccc}
\hline Pathway & Chlorophyll metabolism & Carotenoid biosynthesis \\
\hline all(7005) & 71 & 40 \\
blueviolet(248) & 1 & 2 \\
brown4(236) & 2 & 2 \\
coral(1289) & 20 & 1 \\
darkolivegreen2(1404) & 9 & 6 \\
darkolivegreen4(645) & 1 & 3 \\
honeydew1(274) & 5 & 1 \\
lavenderblush1(1466) & 14 & 14 \\
lightslateblue(7) & 0 & 0 \\
mediumpurple2(682) & 6 & 8 \\
navajowhite(8) & 0 & 0 \\
orange(457) & 6 & 2 \\
plum(42) & 0 & 0 \\
plum1(136) & 3 & 0 \\
sienna4(111) & 4 & 1 \\
\hline
\end{tabular}




\section{Significant Differential Gene Heatmap}

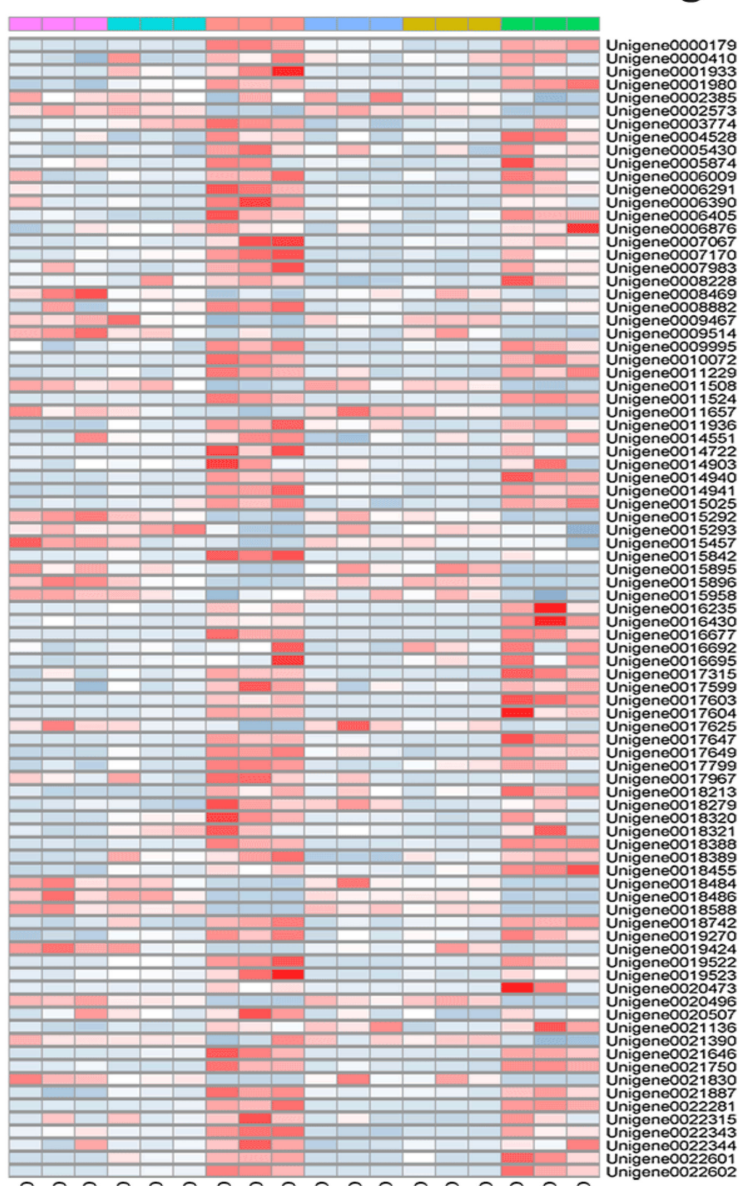

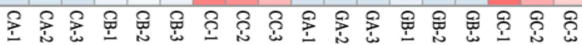

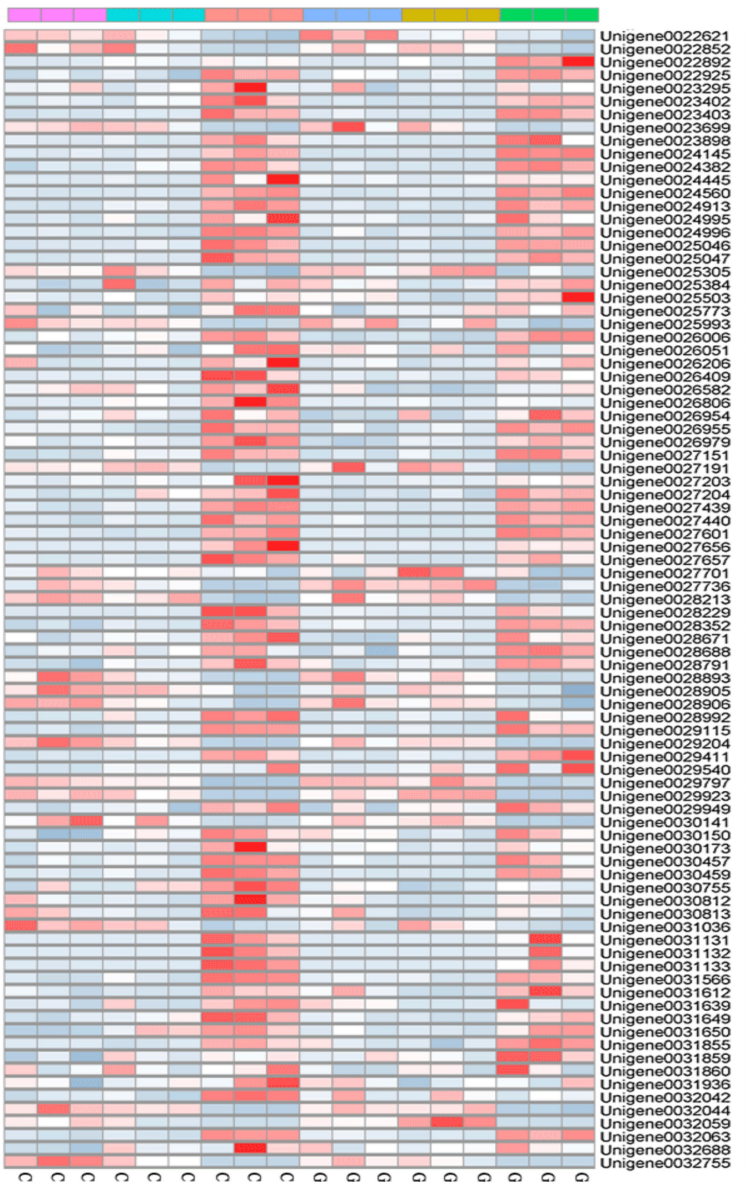

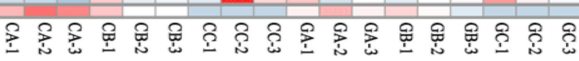

$\begin{array}{lllllllllllllll}3 & 2 & 1 & 0 & -1 & -2 & -3 & \mathrm{CA} & \mathrm{CB} & \mathrm{CC} & \mathrm{GA} & \mathrm{GB} & \mathrm{GC}\end{array}$

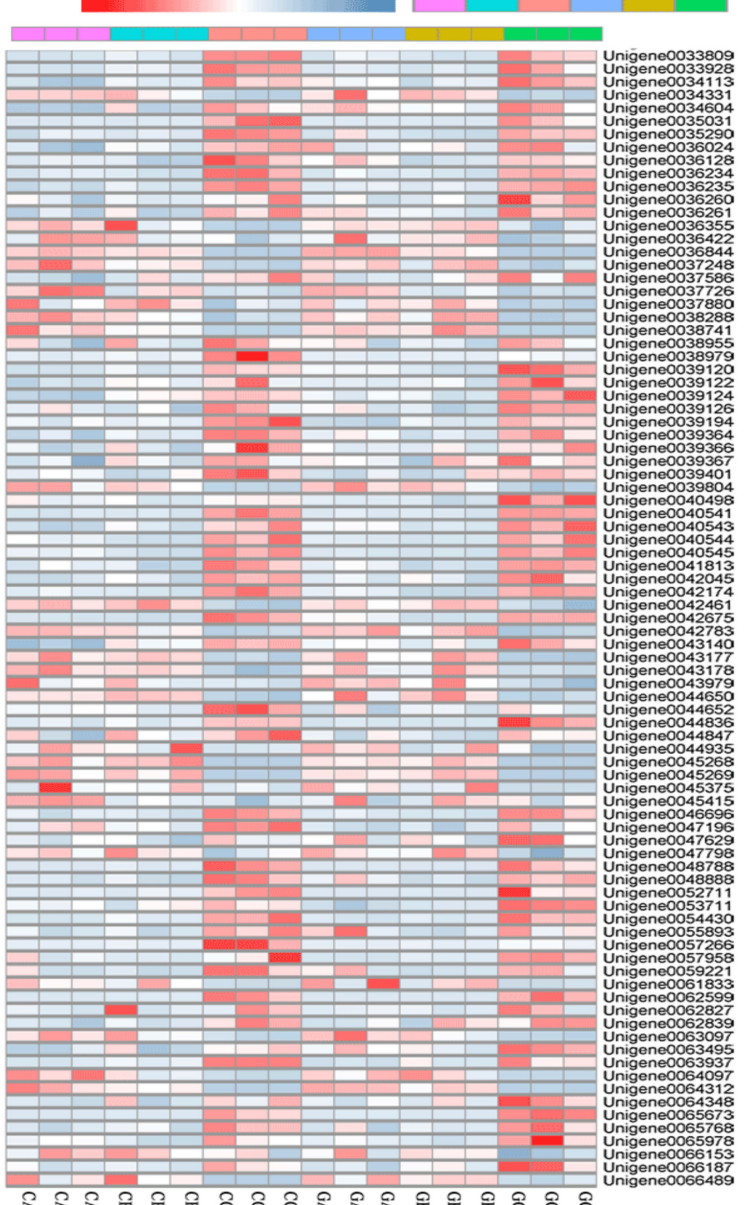

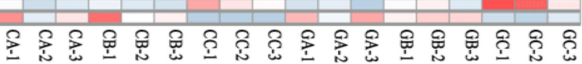

Figure 7. Heatmap of transcription factors in the lavenderblush1 module. The colored leaf in young leaf stage (CA), leaf-expanding stage (CB), and mature leaf stage (CC); the green leaf in young leaf stage (GA), leaf-expanding stage (GB), and mature leaf stage (GC); 1-3: Three biological replicates of each leaf sample. 


\subsection{Co-Expression Networks Reveal a Differential Regulatory Network of Chlorophyll Metabolism and Carotenoid Metabolism}

To understand the regulatory networks of chlorophyll and carotenoid metabolism genes with transcription factors, we first screened 28 related genes in the lavenderblush1 module (Table S7). Then we selected 261 transcription factors from the lavenderblush1 module for Pearson correlation analysis. We plotted all pairs of adjustment relationships using a Pearson correlation coefficient threshold greater than 0.7 (Figure 8). The visualization in Cytoscape showed that there were 180 nodes connected to 1226 edges in the chlorophyll metabolism regulatory networks. There were 170 nodes connected to 922 edges in the carotenoid metabolism regulatory networks. Based on an edge greater than 10, we obtained 5 candidate hub genes for the regulation of chlorophyll and carotenoid metabolism (Table 5).
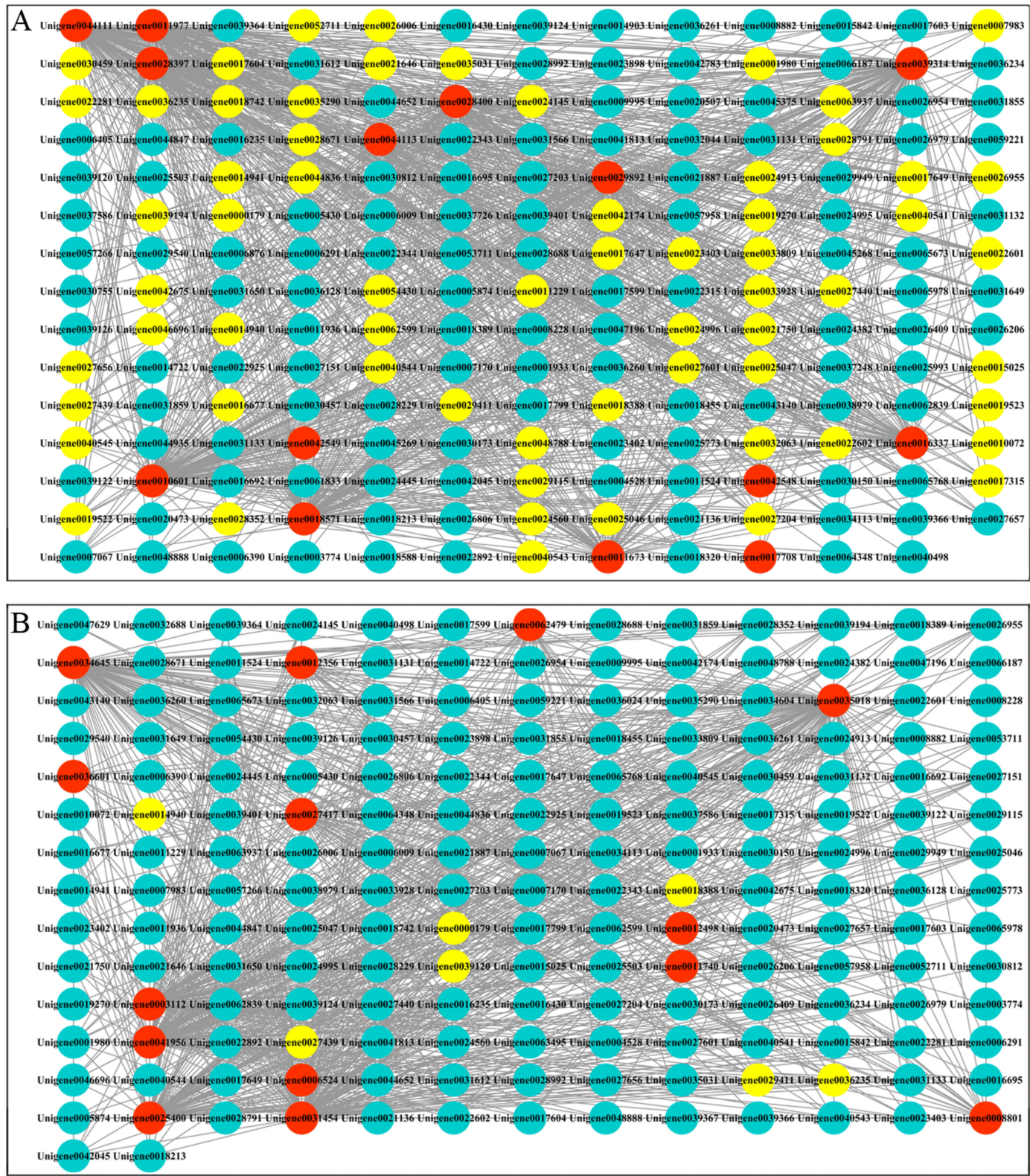

Figure 8. (A) Construction of regulatory networks of chlorophyll metabolism gene and transcription factors in the lavenderblush1 module; (B) Construction of regulatory networks of carotenoid metabolism gene and transcription factors in the lavenderblush1 module. Red nodes indicate structural genes, blue nodes indicate transcription factors, yellow nodes indicate transcription factors with edges greater than 10 . 
Table 5. Candidate hub genes for regulation of chlorophyll metabolism and carotenoid metabolism in the lavenderblush1 modules.

\begin{tabular}{cc}
\hline Gene Name & Descriptions \\
\hline Unigene0018388 & transcription factor TCP20 isoform X2(TCP20) \\
Unigene0027439 & WRKY transcription factor 3(WRKY3) \\
Unigene0036235 & nuclear transcription factor Y subunit C-9-like isoform X1(NFYC9) \\
Unigene0000179 & probable WRKY transcription factor 3 isoform X2(WRKY4) \\
Unigene0029411 & heat stress transcription factor A-7a-like (HSFA2) \\
\hline
\end{tabular}

\subsection{Verification of the Gene Expression through $q R T-P C R$}

In order to further verify the reliability of the transcriptome data, 22 DEGs related to chlorophyll synthesis, photosynthesis, flavonoid synthesis, anthocyanin synthesis, and carotenoid synthesis were selected for qRT-PCR (Figure 9). The expression patterns of 19 genes detected by qRT-PCR were consistent with the transcriptome expression trend during the three stages of leaf color development but Unigene0032350, encoding SGR, Unigene0020260, encoding dihydroflavonol 4-reductase (DFR), and Unigene0044913, encoding UGT75L6, showed different expression trends. The above disparities may have been caused by differences between transcriptome sequencing and qRT-PCR as detection methods, a certain degree of inconsistency (about 30-40\%). The result was normal and reasonable. There was no significant change in gene expression level, resulting in inconsistent detection results. The results showed that the qRT-PCR results of most of the genes were consistent with the transcriptome data.

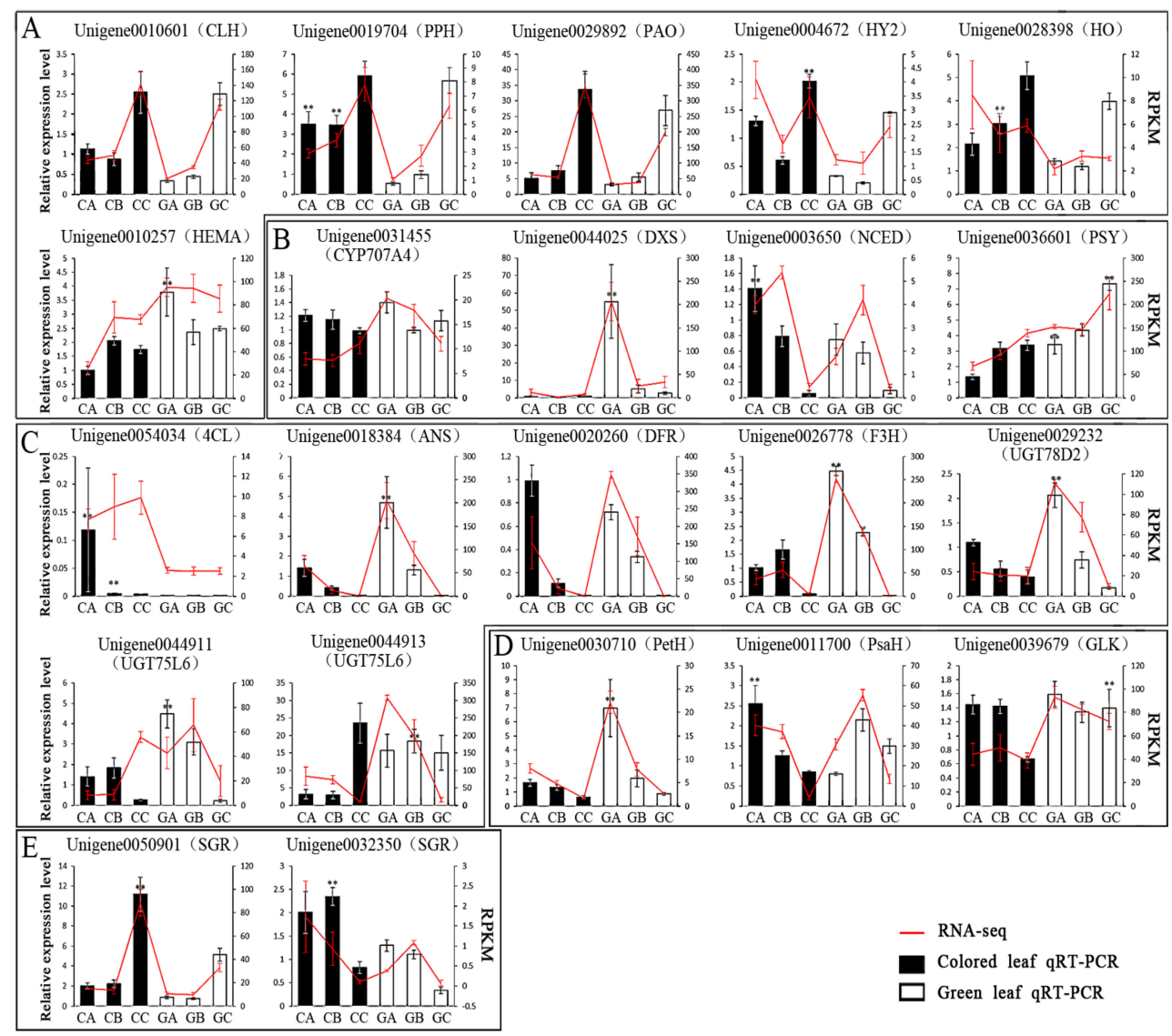

Figure 9. qRT-PCR validation of transcriptome data results for genes associated with leaf color changes. (A) DEGs involved in Porphyrin and chlorophyll metabolism; (B) DEGs involved in Carotenoid biosynthesis; (C) DEGs involved in Biosynthesis of secondary metabolites; (D) DEGs involved in Photosynthesis; (E) DEGs involved in SGR. The significance of differences compared with green leaf is indicated with two asterisks $(p<0.01)$. 


\section{Discussion}

\subsection{Colored Leaves are Closely Related to Chloroplast Dysplasia}

A decrease in the number of chloroplasts and their imperfect development in leaves affect the synthesis of chlorophyll and change leaf color. During the yellowing of Ginkgo biloba leaves, the chloroplast thylakoid membrane layer gradually loosed until the membrane structure gradually disintegrated. The oil particles in the chloroplast increased and eventually disintegrated [27]. The ultrastructure of the colored leaves of Cymbidium sinense [16] and G. biloba [19] mutants was different from that of normal leaves, exhibiting a relatively low number of chloroplasts. The granum thylakoids had a low degree of overlap and loose arrangement, and the membrane structure was fuzzy. The shape was irregular, and the layers were degraded. There were many plastoglobuli but almost no starch grains. The chloroplast ultrastructure of the yellow leaves of Lagerstroemia indica exhibited a ruptured thylakoid membrane and insignificant stromal lamellae [18]. Under natural conditions, the chloroplast granum thylakoids of Camellia sinensis 'Anji Baicha' were poorly developed. The degradation of granum thylakoids, membrane structure, and lamellar structure hindered the biosynthesis of chlorophyll and carotenoids [12].

The results of this study showed that the chloroplast structure of colored and green leaves in $O$. fragrans 'Yinbi Shuanghui' was also significantly different. During the young leaf stage, the chloroplast volume in colored leaves was smaller than that in green leaves. During the mature leaf stage, there were more stromal lamellae in the yellow part of the colored leaves, the granum thylakoids were not stacked normally, and there were a large number of plastoglobuli. The green part of the colored leaf was narrow and small, and the chloroplasts were irregularly long fusiform in shape. The chloroplasts had an unclear outline and no obvious membrane structure. The differences in the structure of these chloroplasts may affect development and photosynthesis in 'Yinbi Shuanghui' leaves, resulting in the inhibition of chlorophyll synthesis and formation of colored leaves.

Chloroplasts use light energy and convert it into chemical energy. GLK, first discovered in Zea mays, was an important transcription factor that affected the development of chloroplasts [28]. Studies in A. thaliana [29], O. sativa [30] and Lycopersicon esculentum [31] found that the expression level of GLK affected the expression of photosynthetic genes and the development of chloroplasts. As in rice, AtGLK1 and AtGLK2 were expressed in partially overlapping domains in photosynthetic tissue. Insertion mutants demonstrate that this expression pattern reflected a degree of functional redundancy, as single mutants display normal phenotypes in most photosynthetic tissues. However, double mutants were pale green in all photosynthetic tissues and chloroplasts exhibited a reduction in granum thylakoids[30] The leaf color mutant of Anthurium andraeanum was accompanied by the downregulation of GLK expression. Ultrastructural observations revealed that chloroplast development was prevented, and the structure was incomplete [32]. The GLK that regulates chloroplast development in the yellow leaves of G. biloba also exhibited downregulated expression [19]. Similarly, the GLK expression in the colored leaves was lower than that of green leaves, and further qRT-PCR also confirmed this result. Consistent with previous studies, the green part of the colored leaf in this study showed few chloroplasts with abnormal development and there were a small number of granum thylakoids and plastoglobuli. This suggests that leaf color variation is closely related to chloroplast dysplasia.

\subsection{Colored Leaves are Affected by the Expression of Genes Related to Chlorophyll Degradation}

Leaf color is affected internally by genetics and externally by environmental factors. It is regulated by leaf cell microstructure and physiological and biochemical metabolic levels. In this study, the colored leaves had a yellow-white margin and green center. Changes in the chlorophyll, carotenoid, and anthocyanin contents of leaves are the main causes of leaf color change. By measuring the chlorophyll and carotenoid contents of leaves in this study, we found that the total chlorophyll and carotenoid content of the colored leaves were significantly lower than that of the green leaves, which led to the phenotypic variation seen in the colored leaves. 
Chlorophyll is an important pigment involved in chloroplast-based photosynthesis. Its function is to capture light energy and drive electron transfer to the reaction center. The entire chlorophyll biosynthesis process requires a 15-step reaction involving 15 enzymes. A number of genes (27 in total) encoding these enzymes have been isolated in the model plant $A$. thaliana [33]. In the chlorophyll biosynthesis pathway, mutations in the coding genes for anyone enzyme may hinder the synthesis of chlorophyll.

First, L-glutamyl-tRNA is converted to $\delta$-aminolevulinic acid (ALA) in a reaction catalyzed by glutamyl-tRNA reductase (GluTR) and glutamate-1-semialdehyde 2,1 aminomutase (GSA). The genes encoding these two enzymes are HEMA and HEML, respectively. ALA is an important precursor substance for chlorophyll synthesis. ALA is converted to porphobilinogen (PBG) in a reaction catalyzed by $\delta$-aminolevulinic acid dehydratase, and then 5 -step biochemical reactions produce protoporphyrin IX (ProtoIX). Therefore, HEMA is a key enzyme gene-regulating chlorophyll synthesis [34] and three HEMA genes have been isolated from A. thaliana, HEMA1, HEMA2, and HEMA3 [35]. Studies have found that the contents of GluTR, ALA, and chlorophyll were all decreased in A. thaliana under HEMA1 gene silencing [36]. In Lagerstroemia indica [18] leaf color mutants, HEMA transcription levels were reduced, with a concurrent decrease in chlorophyll content. In our study, HEMA was significantly downregulated in colored leaves during the young leaf stage and similar results were obtained in qRT-PCR experiments. At the same time, the chlorophyll content in the colored leaves was reduced by $40 \%$ compared with that of the green leaves. Studies indicated that the low expression of HEMA, a key enzyme gene in the chlorophyll synthesis pathway, caused a decrease in enzyme activity and affected chlorophyll synthesis.

Changes in any gene activity during chlorophyll synthesis and degradation may affect the efficiency of these processes. The investigation of chlorophyll degradation in this study focused on the formation of chlorophyllide a (Chlide a) under the action of CLH, followed by a non-enzymatic reaction involving metal-chelating substance (MCS) to remove $\mathrm{Mg}^{2+}$ and form pheophorbide a (Pheide a). The porphyrin ring of Pheide is opened and red chlorophyll metabolite (RCC) produced under the action of PAO. Red chlorophyll metabolite reductase (RCCR) converts it into a primary fluorescent chlorophyll metabolite (pFCC). Finally, non-fluorescent chlorophyll metabolites (NCCs) are produced [35]. CLH has long been recognized as the first enzyme in the chlorophyll degradation pathway. It was first confirmed in Citrus reticulata, and then AtCLH1 and AtCLH2 were isolated from A. thaliana [37]. Harpaz-Saad [38] have suggested that CLH, which catalyzes the hydrolysis of chlorophyll to produce Chlide a, is the rate-limiting enzyme during chlorophyll degradation. In the present study, CLH2 in the colored leaves was significantly upregulated during the young leaf stage. Additionally, the ratio of chlorophyll a to chlorophyll $b$ in the colored leaves was higher than that of the green leaves during the three stages. Therefore, we conclude that the upregulated expression of CLH2 in colored leaves promotes the decomposition of chlorophyll. However, there are other studies that disagree. Following the deletion of two chlorophyllase genes in A. thaliana, leaves were found to senesce normally. It indicated that AtCHL1 and AtCHL2 did not participate in the degradation pathway of chlorophyll [39]. Inhibition of AtCLH1 expression leads to a reduction in the chlorophyll a/b ratio and inhibition of CLH activity but does not affect the rate of chlorophyll degradation during leaf senescence. CLH may, therefore, play a role in the process of converting chlorophyll $b$ at the initial stage of leaf senescence [40]. Therefore, the role of CLH in the chlorophyll degradation pathway remains to be further studied.

In recent years, it has been found that in another pathway of chlorophyll degradation. Chlorophyll a first removes $\mathrm{Mg}^{2+}$ under the action of MCS. The resulting pheophytin a (Phein a) is then removed from the substrate by PPH to form Pheide a[41]. As the amount of $P P H$ expression increased, the rate of Phein a conversion to Pheide a is accelerated, thereby increasing the rate of chlorophyll degradation. Gene expression studies of $C L H$ and $P P H$ during senescence of Brassica oleracea found that cytokinin treatment inhibited the expression of $P P H$, which delayed the degradation of chlorophyll [42]. A small amount of Phein a accumulation was found in the colored leaves of the O. saliva mutant nyc3 and confirmed the presence of the PPH [43]. In this study, Unigene0019704 encoding PPH in the colored 
leaves was upregulated during the three developmental stages. Therefore, we conclude that $P P H$ promoted the degradation of chlorophyll in colored leaves, causing changes in the activity of related enzymes. It may affect the inhibition of chlorophyll synthesis and lead to leaf color variation.

Another key enzyme for chlorophyll degradation is PAO, which catalyzes the decomposition of Phein a to form a colorless substance [44]. Our study showed that during the mature leaf stage, the expression of Unigene0029892, encoding $P A O$, in colored leaves was significantly higher than that in green leaves. Its pattern of expression during leaf color development exhibited an increase with the highest level of expression seen at the mature leaf stage. The slowly increasing expression of $P A O$ in green leaves concurred with the high chlorophyll content in the lavenderblush1 module seen in WGCNA. Previous studies have reported that during the ripening of Ficus carica, the expression of $P A O$ was significantly negatively correlated with chlorophyll levels. Its high level of expression in yellow leaves, therefore, indicated a key role of $P A O$ in degrading chlorophyll in F. carica [45]. The dark induction of senescence in $A$. thaliana leaves showed upregulation of $P A O$ expression and protein levels, and $P A O$ was thought to regulate chlorophyll degradation mainly through its transcription levels [46]. Therefore, the accumulation of high levels of $P A O$ in the mature leaf stage of colored leaves may be the cause of the leaf color phenotype.

The discovery of $S G R$ was a landmark in the study of plant chlorophyll degradation mechanisms. Three SGR homologous proteins, SGR1, SGR2, and SGR-LIKE, were found in the chloroplast vesicles of $A$. thaliana but their roles in chlorophyll degradation were different [47-49]. SGR1 interacts with LHCII protein and CCEs to form an SGR1-CCE-LHCII macromolecular complex that converts Chl to pFCC, thereby accelerating chlorophyll degradation [48]. In natural senescence and dark induction, an SGR1-1 mutant displays a stay-green phenomenon and in SGR1 overexpressing plants, the leaves show yellowing. Both SGR1 and SGRL show specificity in expression. SGR1 (At4g2290) is significantly upregulated during leaf senescence; expression increased rapidly in senescent leaves with a slightly faded green color [49] and the expression of SGR in yellow-leaf Orchid varieties is higher than that in green-leaf varieties [17]. In the present study, seven SGR DEGs were identified. Unigene0050901 encoding $S G R$ was upregulated in colored leaves and most highly expressed during the mature leaf stage, which is consistent with previous studies. We conclude that $S G R$ has a regulatory effect on chlorophyll degradation, resulting in blockage of chlorophyll synthesis.

Transcription factors play an important role in regulating chlorophyll metabolism [50]. In $A$. thaliana, ANAC046, EIN3, and ORE1 affect chlorophyll degradation by regulating the expression of key genes $N Y E 1, N Y C 1$, and $P A O$ in the chlorophyll metabolic pathway [51,52]. Many transcription factors in the NAC and WRKY gene families are also involved in the degradation of chlorophyll [53]. However, the regulatory mechanisms that underlie chlorophyll and carotenoid metabolism in colored leaves of 'Yinbi Shuanghui' have not been revealed. In this study, transcription factors were extracted from the lavenderblush1 module using 28 chlorophyll and carotenoid metabolism-related genes as decoy genes. A total of five genes highly connected with the lavenderblush1 module were identified as candidate hub genes. The major transcription factor families are HSF, NFY, TCP20, and WRKY. To identify putative hub genes, 5 phylogenetic trees were further individually constructed by integration of closely related plant species homologue (Figure S6). In Ginkgo biloba, the DEGs encoding TCP family transcription factors showed significantly differential expression in the golden leaf mutant and may influence leaf coloration [19]. In the present study, Unigene0018388 was grouped into pigment metabolism related-TCP clade, implying that it could also play a crucial role in the regulation of pigment synthesis in 'Yinbi Shuanghui'. These results indicate that the regulatory mechanisms in colored leaves involve complex networks.

\subsection{The Expression of Genes Associated with Carotenoid Metabolism in Colored Leaves impedes the Synthesis of Carotenoids}

Carotenoids are found mainly in plant chloroplasts and many flowers and fruits. They also play important roles in plant photosynthesis. The precursor of carotenoid biosynthesis is isoprene 
pyrophosphate (IPP), which is synthesized by the 2-C-methyl-D-erythritol-4-phosphate (MEP) pathway in the plastid. DXS plays a key role in the regulation of terpenoid synthesis and is the first and rate-limiting enzyme in the MEP pathway. The enzyme is localized in the thylakoids. Excessive expression of DXS2 can effectively promote the accumulation of carotenoids and isoprene [54]. A significant increase in chlorophyll and carotene in leaves of $A$. thaliana overexpressing the StDXS1 gene [55]. Carotenoids and chlorophyll and other endogenous hormones (such as ABA) in Daucus carota and A. thaliana transformed with the DXS are increased [56]. The synthesis of carotenoids starts with geranylgeranyl diphosphate (GGPP) and produces colorless phytoene under the action of PSY. PSY is a key regulatory enzyme that determines the total amount of carotenoid accumulation in plants [57]. The relative expression of PSY in Capsicum annuum and Actinidia macrosperma was high and the carotenoid content was also increased [58,59]. In transgenic plants of Lycopersicon esculentum, overexpression of PSY resulted in a significant increase in carotenoid content in the seed coat, cotyledon, and hypocotyl [60]. In this study, the genes encoding DXS and PSY in colored leaves showed significant downregulation during the three stages of leaf development, but PSY expression was seen gradually to increase. qRT-PCR further confirmed the presence of the DXS and PSY. Their expression in colored leaves was lower than that in green leaves throughout development. In addition, the PSY was enriched in the lavenderblush1 module in WGCNA, indicating that PSY affects the carotenoid synthesis and causes a decrease in carotenoid accumulation.

Carotenoid degradation also affects the carotenoid content. There are two main carotenoid cleavage pathways in plants, which involved carotenoid dioxygenase (CCD) and NCED [61]. NCEDs are rate-limiting enzymes that control the conversion of carotenoids to ABA by catalyzing the formation of the ABA precursor xanthophyll [62]. Nine NCED have been identified in A. thaliana, of which five are in plastids. The regulation of AtNCED3 can affect the carotenoid content of the plastid membrane [63]. For example, during the process of peony leaf color change, the NCED was upregulated, resulting in a decrease in carotenoid accumulation [64]. Our study found that the gene encoding NCED was upregulated in colored leaves and the carotenoid content in these leaves was significantly lower than that in green leaves during the mature leaf stages. The results are consistent with those found in other leaf color mutants. We conclude that the upregulated expression of NCED promotes the degradation of carotenoids, resulting in a decrease in carotenoid content in the colored leaves.

\section{Conclusions}

In our study, the physiological and transcriptome sequences of colored and green leaves of $O$. fragrans 'Yinbi Shuanghui' were studied at different developmental stages. Low chlorophyll content and abnormal chloroplast structure were observed in colored leaves. Transcriptome data identified DEGs and pathways related to chlorophyll and carotenoid metabolism. In addition, the association between the co-expressed gene modules formed by DEGs and the physiological indicators was analyzed by WGCNA. Five hub genes involved in regulating chlorophyll and carotenoid metabolism were identified. Consequently, the gene expression related to chlorophyll degradation, carotenoid synthesis, and GLK may be related to the formation of colored leaves. These genes would be taken as the candidate genes for our future transgenic improvement. Our research will supply a valuable gene resource for guided plant breeding.

Supplementary Materials: The following are available online at http://www.mdpi.com/2218-273X/10/4/549/s1, Table S1: Primer sequences used for qRT-PCR, Table S2: Top 20 candidate genes among the annotated DEGs of colored and green leaf in different stages, Table S3: Metabolic pathways in which DEGs are significantly enriched in CA vs. GA, Table S4: Metabolic pathways in which DEGs are significantly enriched in CB vs. GB, Table S5: Metabolic pathways in which DEGs are significantly enriched in CC vs. GC, Table S6: Annotation of transcription factors in lavenderblush1 module, Table S7: 28 chlorophyll metabolism- and carotenoid metabolism-related genes in the lavenderblush1 module, Figure S1: PCA of all samples, Figure S2: Volcano plots of differential gene expression of all samples in different leaf stages, Figure S3: Module hierarchical clustering tree, Figure S4: Classification of transcription factors in the lavenderblush1 module, Figure S5: The cluster of different bioreps in Figure 7; Figure S6: Phylogenetic tree derived from amino acid sequences of 5 hub genes. 
Author Contributions: Conceptualization, L.W., Y.Y. and X.Y.; validation, X.C. and J.X.; methodology, W.D. and Y.L.; writing-original draft, X.C.; writing-review and editing, X.C. and Y.Y; project administration, X.C. and L.W. All authors have read and agreed to the published version of the manuscript.

Funding: This work was supported by the National Natural Science Foundation of China (Grant Nos. 31870695 and 31601785), Science and Technology Plan Project of Jiangsu Province (BE2017375), the Priority Academic Program Development of Jiangsu Higher Education Institutions (PAPD); the Natural Science Foundation of the Jiangsu Higher Education Institutions of China (No. 19KJB220012)

Acknowledgments: We are grateful to Gene Denovo for technical support.

Conflicts of Interest: The authors declare no conflict of interest.

\section{References}

1. Kang, S.J.; Xia, F.Y.; Xing., Z.G.; Pu., R.B.; Juan., Z.; Jun., D.G.; Xian., Y.M.; Yu., G.Z.; Li, Z. Gene Encoding Protochlorophyllide Oxidoreductase B Is Involved in Chlorophyll Synthesis of Rice. Crop. Sci. 2015, 55, 284-293. [CrossRef]

2. Guo, H.J.; Zhao, H.B.; Zhao, L.S.; Gu, J.Y.; Zhao, S.R.; Li, J.H.; Liu, Q.C.; Liu, L.X. Characterization of a Novel Chlorophyll-Deficient Mutant Mt6172 in Wheat. J.Integr. Agr. 2012, 11, 888-897. [CrossRef]

3. Waters, M.T.; Wang, P.; Korkaric, M.; Capper, R.G.; Saunders, N.J.; Langdale, J.A. GLK Transcription Factors Coordinate Expression of the Photosynthetic Apparatus in Arabidopsis. Plant Cell 2009, 21, 1109-1128. [CrossRef] [PubMed]

4. Chu, P.; Yan, G.X.; Yang, Q.; Zhai, L.N.; Zhang, C.; Zhang, F.Q.; Guan, R.Z. iTRAQ-based quantitative proteomics analysis of Brassica napus leaves reveals pathways associated with chlorophyll deficiency. $J$. Proteomics 2015, 113, 244-259. [CrossRef] [PubMed]

5. Lu, M.; Liu, H.H.; Mao, H.; Zhao, Q.R.; Zhao, H.X.; Hu, S.W. Chlorophyll anabolic changes in leaves of Brassica napus L. yellow mutants. Acta Botanica Boreali Occidential Sinica 2010, 30, 2177-2183.

6. Ou, L.J. Study on the development of chloroplasts in rice leaf color mutants. Acta Botanica Boreali Occidential Sinica 2010, 30, 85-92.

7. Lu, D.H.; Zong, X.F.; Wang, S.G.; Ling, Y.H.; Sang, X.C.; Hua, H.G. Photosynthetic characteristics of two rice leaf color mutants. Crop. J. 2009, 35, 2304-2308.

8. Zhou, H.; Pan, Y.Z.; Liu, X.Z.; Ma, X.J.; Chen, S.L.; Lin, D.Z.; Wang, J.M.; Dong, Y.J.; Teng, S. Genetic analysis of a new rice chlorophyll-deficient yellow leaf mutant and its gene mapping. Mol. Plant Breed. 2013, 11, $145-151$.

9. Ma, X.Z.; Sun, X.Q.; Li, C.M.; Huan, R.; Sun, C.H.; Wang, Y.; Xiao, F.L.; Wang, Q.; Chen, P.R.; Ma, F.R.; et al. Map-based cloning and characterization of the novel yellow-green leaf gene ys 83 in rice (Oryza sativa). Plant Physiol. Biochem. 2017, 111, 1-9. [CrossRef]

10. Zhu, X.; Guo, S.; Wang, Z.; Du, Q.; Xing, Y.; Zhang, T.; Shen, W.; Sang, X.; Ling, Y.; He, G. Map-based cloning and functional analysis of YGL8, which controls leaf colour in rice (Oryza sativa). BMC Plant Biol. 2016, 16, 134. [CrossRef]

11. Gomez, F.; Carrión, C.; Costa, M.; Desel, C.; Kieselbach, T.; Funk, C.; Krupinska, K.; Guiamet, J. Extra-plastidial degradation of chlorophyll and photosystem I in tobacco leaves involving "senescence-associated vacuoles". Plant J. 2019, 99, 465-477. [CrossRef] [PubMed]

12. Li, C.F.; Xu, Y.X.; Ma, J.Q.; Jin, J.Q.; Huang, D.J.; Yao, M.Z.; Ma, C.L.; Chen, L. Biochemical and transcriptomic analyses reveal different metabolite biosynthesis profiles among three color and developmental stages in 'Anji Baicha' (Camellia sinensis). BMC Plant Biol. 2016, 16, 195. [CrossRef]

13. Lu, M.; Han, J.; Zhu, B.; Jia, H.; Yang, T.; Wang, R.; Deng, W.W.; Zhang, Z.Z. Significantly increased amino acid accumulation in a novel albino branch of the tea plant (Camellia sinensis). Planta 2019, 249, 363-376. [CrossRef] [PubMed]

14. Wang, L.; Yue, C.; Cao, H.L.; Zhou, Y.H.; Zeng, J.M.; Yang, Y.J.; Wang, X.H. Biochemical and transcriptome analyses of anovel chlorophyll-deficient chlorina tea plant cultivar. BMC Plant Biol. 2014, 14, 352. [CrossRef] [PubMed]

15. Wu, H.Y.; Shi, N.R.; An, X.Y.; Liu, C.; Fu, H.F.; Cao, L.; Feng, Y.; Sun, D.J.; Zhang, L.L. Candidate Genes for Yellow Leaf Color in Common Wheat (Triticum aestivum L.) and Major Related Metabolic Pathways according to Transcriptome Profiling. Int. J. Mol. Sci. 2018, 19, 1594. [CrossRef] [PubMed] 
16. Zhu, G.; Yang, F.; Shi, S.; Li, D.; Wang, Z.; Liu, H.; Huang, D.; Wang, C. Transcriptome Characterization of Cymbidium sinense 'Dharma' Using 454 Pyrosequencing and Its Application in the Identification of Genes Associated with Leaf Color Variation. PLoS ONE 2015, 10, e0128592. [CrossRef] [PubMed]

17. Jiang, Y.; Song, H.Y.; He, J.R.; Wang, Q.; Liu, J. Comparative transcriptome analysis provides global insight into gene expression differences between two orchid cultivars. PLoS ONE 2018, 13, e0200155. [CrossRef]

18. Li, Y.; Zhang, Z.Y.; Wang, P.; Wang, S.A.; Ma, L.L.; Li, L.F.; Yang, R.T.; Ma, Y.Z.; Wang, Q. Comprehensive transcriptome analysis discovers novel candidate genes related to leaf color in a Lagerstroemia indica yellow leaf mutant. Genes Genomics 2015, 37, 851-863. [CrossRef]

19. Li, W.; Yang, S.; Lu, Z.; He, Z.; Ye, Y.; Zhao, B.; Wang, L.; Jin, B. Cytological, physiological, and transcriptomic analyses of golden leaf coloration in Ginkgo biloba L. Hortic. Res. 2018, 5, 12. [CrossRef]

20. Mu, H.N.; Li, H.G.; Wang, L.G.; Yang, X.L.; Sun, T.Z.; Xu, C. Transcriptome sequencing and analysis of sweet osmanthus (Osmanthus fragrans Lour.). Genes Genomics 2014, 36, 777-788. [CrossRef]

21. Xiang, M.; Duan, Y.F.; Xiang, Q.B. Establishment of Caiyegui Group. J. Nanjing For. Univ. Nat. Sci. Ed. 2014, 38, 187.

22. Zhang, X.Z. Comparative study on determination methods of plant chlorophyll content. J. Shenyang Agr. Univ. 1985, 4, 84-87.

23. Zhang, C.; Fu, J.X.; Wang, Y.G.; Bao, Z.Y.; Zhao, H.B. Identification of Suitable Reference Genes for Gene Expression Normalization in the Quantitative Real-Time PCR Analysis of Sweet Osmanthus (Osmanthus fragrans Lour.). PLoS ONE 2015, 10, e0136355. [CrossRef] [PubMed]

24. Qiu, Z.; Chen, D.; He, L.; Zhang, S.; Yang, Z.; Zhang, Y.; Wang, Z.; Ren, D.; Qian, Q.; Guo, L.; et al. The rice white green leaf 2 gene causes defects in chloroplast development and affects the plastid ribosomal protein S9. Rice 2018, 11, 39. [CrossRef]

25. Mansilla, N.; Racca, S.; Gras, D.; Gonzalez, D.; Welchen, E. The Complexity of Mitochondrial Complex IV: An Update of Cytochrome c Oxidase Biogenesis in Plants. Int. J. Mol. Sci. 2018, 19, 662. [CrossRef]

26. Zhao, X.; Feng, B.H.; Chen, T.T.; Zhang, C.X.; Tao, L.X.; Fu, G.F. Transcriptome analysis of pale-green leaf rice reveals photosynthetic regulatory pathways. Acta Physiologiae Plantarum 2017, 39, 274. [CrossRef]

27. Wei, X.D.; Chen, G.X.; Xu, Y.L.; Lei, H.; Shi, D.W. Changes of Photosynthetic Physiological Characteristics and Chloroplast Ultrastructure of Ginkgo Leaves During Senescence. Bull. Botan. Res. 2008, 04, 433-437.

28. Hall, L.N.; Rossini, L.; Cribb, L.; Langdale, J.A. GOLDEN 2: A novel transcriptional regulator of cellular differentiation in the maize leaf. Plant Cell 1998, 10, 925-936. [CrossRef]

29. Waters, M.T.; Moylan, E.C.; Langdale, J.A. GLK transcription factors regulate chloroplast development in a cell-autonomous manner. Plant J. 2008, 56, 432-444. [CrossRef]

30. Fitter, D.W.; Martin, D.J.; Copley, M.J.; Scotland, R.W.; Langdale, J.A. GLK gene pairs regulate chloroplast development in diverse plant species. Plant J. 2002, 31, 713-727. [CrossRef]

31. Powell, A.L.T.; Nguyen, C.V.; Hill, T.; Cheng, K.L.; Figueroa-Balderas, R.; Aktas, H.; Ashrafi, H.; Pons, C.; Fernandez-Munoz, R.; Vicente, A.; et al. Uniform ripening Encodes a Golden 2-like Transcription Factor Regulating Tomato Fruit Chloroplast Development. Science 2012, 336, 1711-1715. [CrossRef] [PubMed]

32. Yang, M.; Zhu, L.; Pan, C.; Xu, L.; Liu, Y.; Ke, W.; Yang, P. Transcriptomic Analysis of the Regulation of Rhizome Formation in Temperate and Tropical Lotus (Nelumbo nucifera). Sci. Rep. 2015, 5, 13059. [CrossRef] [PubMed]

33. Nagata, N.; Tanaka, R.; Satoh, S.; Tanaka, A. Identification of a vinyl reductase gene for chlorophyll synthesis in Arabidopsis thaliana and implications for the evolution of Prochlorococcus species. Plant Cell 2005, 17, 233-240. [CrossRef] [PubMed]

34. Liu, W.; Fu, Y.; Hu, G.; Si, H.; Zhu, L.; Wu, C.; Sun, Z. Identification and fine mapping of a thermo-sensitive chlorophyll deficient mutant in rice (Oryza sativa L.). Planta 2007, 226, 785-795. [CrossRef] [PubMed]

35. Hoertensteiner, S. Update on the biochemistry of chlorophyll breakdown. Plant Mol. Biol. 2013, 82, 505-517. [CrossRef] [PubMed]

36. Kumar, A.M.; Soll, D. Antisense HEMA1 RNA expression inhibits heme and chlorophyll biosynthesis in arabidopsis. Plant Physiol. 2000, 122, 49-56. [CrossRef]

37. Tsuchiya, T.; Ohta, H.; Okawa, K.; Iwamatsu, A.; Shimada, H.; Masuda, T.; Takamiya, K. Cloning of chlorophyllase, the key enzyme in chlorophyll degradation: Finding of a lipase motif and the induction by methyl jasmonate. Proc. Natl. Acad. Sci. USA 1999, 96, 15362-15367. [CrossRef] 
38. Harpaz Saad, S.; Azoulay, T.; Arazi, T.; Ben-Yaakov, E.; Mett, A.; Shiboleth, Y.M.; Hoertensteiner, S.; Gidoni, D.; Gal-On, A.; Goldschmidt, E.E.; et al. Chlorophyllase is a rate-limiting enzyme in chlorophyll catabolism and is posttranslationally regulated. Plant Cell 2007, 19, 1007-1022. [CrossRef]

39. Schenk, N.; Schelbert, S.; Kanwischer, M.; Goldschmidt, E.E.; Doermann, P.; Hoertensteiner, S. The chlorophyllases AtCLH1 and AtCLH2 are not essential for senescence-related chlorophyll breakdown in Arabidopsis thaliana. FEBS Lett. 2007, 581, 5517-5525. [CrossRef]

40. Zhou, X.; Liao, Y.; Ren, G.D.; Zhang, Y.Y.; Chen, W.J.; Kuai, B.K. Repression of AtCLH1 expression results in a decrease in the ratio of chlorophyll $\mathrm{a} / \mathrm{b}$ but doesnot affect the rate of chlorophyll degradation during leaf senescence. J. Plant Physiol. Mol. Biol. 2007, 33, 596-606.

41. Suzuki, T.; Kunieda, T.; Murai, F.; Morioka, S.; Shioi, Y. Mg-dechelation activity in radish cotyledons with artificial and native substrates, Mg-chlorophyllin a and chlorophyllide a. Plant Physiol. Biochem. 2005, 43, 459-464. [CrossRef] [PubMed]

42. Buechert, A.M.; Civello, P.M.; Martinez, G.A. Chlorophyllase versus pheophytinase as candidates for chlorophyll dephytilation during senescence of broccoli. J. Plant Physiol. 2011, 168, 337-343. [CrossRef]

43. Morita, R.; Sato, Y.; Masuda, Y.; Nishimura, M.; Kusaba, M. Defect in non-yellow coloring 3, an alpha/beta hydrolase-fold family protein, causes a stay-green phenotype during leaf senescence in rice. Plant J. 2009, 60, 1110.

44. Pruzinska, A.; Tanner, G.; Anders, I.; Roca, M.; Hortensteiner, S. Chlorophyll breakdown: Pheophorbide a oxygenase is a Rieske-type iron-sulfur protein, encoded by the accelerated cell death 1 gene. Proc. Natl. Acad. Sci. USA 2003, 100, 15259-15264. [CrossRef] [PubMed]

45. Rosianskey, Y.; Dahan, Y.; Yadav, S.; Freiman, Z.E.; Milo-Cochavi, S.; Kerem, Z.; Eyal, Y.; Flaishman, M.A. Chlorophyll metabolism in pollinated vs. parthenocarpic fig fruits throughout development and ripening. Planta 2016, 244, 491-504. [CrossRef] [PubMed]

46. Pruzinska, A.; Tanner, G.; Aubry, S.; Anders, I.; Moser, S.; Muller, T.; Ongania, K.H.; Krautler, B.; Youn, J.Y.; Liljegren, S.J.; et al. Chlorophyll breakdown in senescent Arabidopsis leaves. Characterization of chlorophyll catabolites and of chlorophyll catabolic enzymes involved in the degreening reaction. Plant Physiol. 2005, 139, 52-63. [CrossRef]

47. Mecey, C.; Hauck, P.; Trapp, M.; Pumplin, N.; Plovanich, A.; Yao, J.; He, S.Y. A Critical Role of STAYGREEN/Mendel's I Locus in Controlling Disease Symptom Development during Pseudomonas syringae pv tomato Infection of Arabidopsis. Plant Physiol. 2011, 157, 1965-1974. [CrossRef]

48. Sakuraba, Y.; Schelbert, S.; Park, S.Y.; Han, S.H.; Lee, B.D.; Andres, C.B.; Kessler, F.; Hoertensteiner, S.; Paek, N.C. STAY-GREEN and Chlorophyll Catabolic Enzymes Interact at Light-Harvesting Complex II for Chlorophyll Detoxification during Leaf Senescence in Arabidopsis. Plant Cell 2012, 24, 507-518. [CrossRef]

49. Ren, G.D.; An, K.; Liao, Y.; Zhou, X.; Cao, Y.J.; Zhao, H.F.; Ge, X.C.; Kuai, B.K. Identification of a novel chloroplast protein AtNYE1 regulating chlorophyll degradation during leaf senescence in arabidopsis. Plant Physiol. 2007, 144, 1429-1441. [CrossRef]

50. Lin, M.; Pang, C.Y.; Fan, S.L.; Song, M.Z.; Wei, H.L.; Yu, S.X. Global analysis of the Gossypium hirsutum L. Transcriptome during leaf senescence by RNA-Seq. BMC Plant Biol. 2015, 15, 43. [CrossRef]

51. Qiu, K.; Li, Z.P.; Yang, Z.; Chen, J.Y.; Wu, S.X.; Zhu, X.Y.; Gao, S.; Gao, J.; Ren, G.D.; Kuai, B.K.; et al. EIN3 and ORE1 Accelerate Degreening during Ethylene-Mediated Leaf Senescence by Directly Activating Chlorophyll Catabolic Genes in Arabidopsis. PLoS Genet. 2015, 11, e1005399. [CrossRef] [PubMed]

52. Oda-Yamamizo, C.; Mitsuda, N.; Sakamoto, S.; Ogawa, D.; Ohme-Takagi, M.; Ohmiya, A. The NAC transcription factor ANAC 046 is a positive regulator of chlorophyll degradation and senescence in Arabidopsis leaves. Sci. Rep. 2016, 6, 23609. [CrossRef] [PubMed]

53. Wen, C.H.; Lin, S.S.; Chu, F.H. Transcriptome Analysis of a Subtropical Deciduous Tree: Autumn Leaf Senescence Gene Expression Profile of Formosan Gum. Plant Cell Physiol. 2015, 56, 163-174. [CrossRef]

54. Ohmiya, A.; Kishimoto, S.; Aida, R.; Yoshioka, S.; Sumitomo, K. Carotenoid cleavage dioxygenase (CmCCD4a) contributes to white color formation in chrysanthemum petals. Plant Physiol. 2006, 142, 1193-1201. [CrossRef] [PubMed]

55. Henriquez, M.A.; Soliman, A.; Li, G.; Hannoufa, A.; Ayele, B.T.; Daayf, F. Molecular cloning, functional characterization and expression of potato (Solanum tuberosum) 1-deoxy-D-xylulose 5-phosphate synthase 1 (StDXS1) in response to Phytophthora infestans. Plant. Sci. 2016, 243, 71-83. [CrossRef] [PubMed] 
56. Simpson, K.; Quiroz, L.F.; Rodriguez-Concepcion, M.; Stenge, C.R. Differential Contribution of the First Two Enzymes of the MEP Pathway to the Supply of Metabolic Precursors for Carotenoid and Chlorophyll Biosynthesis in Carrot (Daucus carota). Front. Plant Sci. 2016, 7, 1344. [CrossRef]

57. Burkhardt, P.K.; Beyer, P.; Wunn, J.; Kloti, A.; Armstrong, G.A.; Schledz, M.; von Lintig, J.; Potrykus, I. Transgenic rice (Oryza sativa) endosperm expressing daffodil (Narcissus pseudonarcissus) phytoene synthase accumulates phytoene, a key intermediate of provitamin A biosynthesis. Plant J. 1997, 11, 1071-1078. [CrossRef]

58. Ampomah Dwamena, C.; McGhie, T.; Wibisono, R.; Montefiori, M.; Hellens, R.P.; Allan, A.C. The kiwifruit lycopene beta-cyclase plays a significant role in carotenoid accumulation in fruit. J. Exp. Bot. 2009, 60, 3765-3779. [CrossRef]

59. Ha, S.H.; Kim, J.B.; Park, J.S.; Lee, S.W.; Cho, K.J. A comparison of the carotenoid accumulation in Capsicum varieties that show different ripening colours: Deletion of the capsanthin-capsorubin synthase gene is not a prerequisite for the formation of a yellow pepper. J. Exp. Bot. 2007, 58, 3135-3144. [CrossRef]

60. Fray, R.G.; Grierson, D. Identification and genetic analysis of normal and mutant phytoene synthase genes of tomato by sequencing, complementation and co-suppression. Plant Mol. Biol. 1993, 22, 589-602. [CrossRef]

61. Ruiz-Sola, M.A.; Rodriguez-Concepcion, M. Carotenoid biosynthesis in Arabidopsis: A colorful pathway. Arabidopsis B. Am. Soc. Plant. Biol. 2012, 10, e0158. [CrossRef] [PubMed]

62. Nambara, E.; Marion-Poll, A. Abscisic acid biosynthesis and catabolism. Annu. Rev. Plant. Biol. 2005, 56, 165-185. [CrossRef] [PubMed]

63. Tan, B.C.; Joseph, L.M.; Deng, W.T.; Liu, L.J.; Li, Q.B.; Cline, K.; McCarty, D.R. Molecular characterization of the Arabidopsis 9-cis epoxycarotenoid dioxygenase gene family. Plant J. 2003, 35, 44-56. [CrossRef] [PubMed]

64. Luo, J.; Duan, J.; Huo, D.; Shi, Q.; Niu, L.; Zhang, Y. Transcriptomic Analysis Reveals Transcription Factors Related to Leaf Anthocyanin Biosynthesis in Paeonia qiui. Molecules 2017, 22, 2186. [CrossRef] [PubMed]

(C) 2020 by the authors. Licensee MDPI, Basel, Switzerland. This article is an open access article distributed under the terms and conditions of the Creative Commons Attribution (CC BY) license (http://creativecommons.org/licenses/by/4.0/). 